Rabaska

Revue d'ethnologie de l'Amérique française

\title{
Les Trois Bérets et les ateliers de sculpture de
}

Saint-Jean-Port-Joli, 1930-1967

The Three Berets and the Sculpture Workshops of

Saint-Jean-Port-Joli, 1930-1967

\section{Jean-François Blanchette}

Volume 18, 2020

URI : https://id.erudit.org/iderudit/1072900ar

DOI : https://doi.org/10.7202/1072900ar

Aller au sommaire du numéro

Éditeur(s)

Société québécoise d'ethnologie

ISSN

1703-7433 (imprimé)

1916-7350 (numérique)

Découvrir la revue

Citer cet article

Blanchette, J.-F. (2020). Les Trois Bérets et les ateliers de sculpture de Saint-Jean-Port-Joli, 1930-1967. Rabaska, 18, 11-42.

https://doi.org/10.7202/1072900ar
Résumé de l'article

Les frères Bourgault, Médard (1897-1967), André (1898-1958) et Jean-Julien (1910-1996), connus sous le nom des Trois Bérets, ont été à l'origine d'un mouvement esthétique singulier, authentique et identitaire. Ils ont formé des centaines d'apprentis à la sculpture sur bois en taille directe. L'examen de l'organisation de leurs ateliers permet de réaliser qu'ils ont tenu à bout de bras leurs entreprises, avec peu d'aide externe si ce n'est l'encouragement des clients souvent issus de l'élite. Le paradigme de l'apprentissage à l'école est remplacé par la pratique en atelier avec le maître. 


\title{
Études
}

\section{2]. \\ Les Trois Bérets et les ateliers de sculpture de Saint-Jean-Port-Joli, 1930-1967}

\author{
Jean-François Blanchette \\ Société québécoise d'ethnologie
}

\section{Introduction}

Les trois frères Bourgault, Médard (1897-1967), André (1898-1958) et Jean-Julien (1910-1996), communément connus sous le nom des Trois Bérets, en raison de leur couvre-chef, ont été à l'origine d'un mouvement de sculpture sur bois en taille directe qui a fait vivre à une certaine époque plus d'une centaine de familles. Les trois frères ont formé de nombreux apprentis dont plusieurs sont devenus maîtres d'atelier eux-mêmes. On peut évaluer qu'un millier de sculpteurs ont été formés et ont travaillé dans le sillon des Trois Bérets. C'est donc avec fierté pour tout le village que Saint-Jean-Port-Joli soit devenu la capitale de la sculpture sur bois. Le présent article se veut une brève présentation des ateliers des trois frères et de leur impact sur ce mouvement de sculpture sur bois totalement unique en Amérique du Nord ${ }^{1}$.

Je désire, au cours de cet article, donner la parole aux sculpteurs ${ }^{2}$ et mettre en évidence la singularité du mouvement de sculpture sur bois de Saint-Jean-Port-Joli, un mouvement esthétique authentique et identitaire. Le paradigme de la formation à l'époque mettait l'accent sur le terme école pour définir un lieu d'apprentissage et on a étiqueté les ateliers avec ce terme, ce qui, aujourd'hui, ne permet pas de saisir toute la nuance et l'importance de la formation totalement originale qu'on y trouvait. En effet, ce n'est qu'à partir de 1967, lorsque l'École de sculpture Jean-J. Bourgault deviendra l'École de sculpture de Saint-Jean-Port-Joli avec son

1. Une demande d'identification des trois frères Bourgault a été présentée à la municipalité de Saint-Jean-Port-Joli par la Société québécoise d'ethnologie le 6 janvier 2020. Le document, rédigé par Suzanne Marchand à partir de recherches effectuées par Jean-François Blanchette, Jean Simard et ellemême sera publiée sur le site de la SQE (ethnologiequebec.org) au cours de l'année 2020.

2. Grâce aux riches entrevues faites avec eux par divers intervenants et déposées au Musée de la mémoire vivante (désormais MMv). J'aimerais remercier André-Médard, Nicole et Pierre Bourgault pour leur chaleureuse contribution aux entrevues que j'ai réalisées avec eux. 
nouveau directeur Pierre Bourgault ${ }^{3}$, sous l'égide de la Commission scolaire régionale Pascal-Taché, qu'il y aura un curriculum bien défini en lien avec un programme scolaire ${ }^{4}$. Jusqu'en 1967, en effet, il n'y a pas de curriculum ni d'enseignement magistral dans les ateliers de sculpture de Saint-JeanPort-Joli. On apprend par la pratique avec le maître. La sculpture sur bois en taille directe ${ }^{5}$ est la technique privilégiée. Nul n'est besoin de faire un dessin élaboré ou de créer un modèle d'argile au préalable. C'est une approche qui est familière aux sculpteurs d'art populaire traditionnel québécois et qui caractérise les ateliers de Saint-Jean-Port-Joli.

\section{Au début}

Les trois frères, nés à Saint-Jean-Port-Joli, sont les enfants de Magloire Bourgault, menuisier, et d'Émélie Legros, modiste et mère d'une famille qui en comptera seize. C'est en jouant dans l'atelier de leur père que les enfants, filles comme garçons, apprennent le sens du bois. Saint-Jean-Port-Joli est alors un village de cultivateurs, de marins et d'artisans, comme de nombreux autres villages riverains de la Côte-du-Sud. Comme le territoire agraire est limité, le fleuve attire de nombreux jeunes hommes qui deviennent marins. C'est le cas de Médard, d'André et de Jean-Julien. Mais le premier se démarque. Quand ses compagnons de voyage profitent d'un arrêt pour aller prendre un verre, il achète des livres et retourne lire dans sa cabine ${ }^{6}$.

C'est l'époque des mains habiles où on fabrique avec aisance des objets avec les ressources disponibles. L'art populaire traditionnel est prégnant dans les communautés rurales. À l'église de Saint-Jean-Port-Joli, où Médard aime aller se recueillir, l'art des grands artistes québécois comme Pierre-Noël Levasseur, Jean Baillairgé et ses fils Pierre-Florent et François ${ }^{7}$ inspire les habitants. L'art populaire est aussi présent dans l'église grâce aux maquettes de bateaux qui sont suspendues au plafond en guise d'ex-voto ${ }^{8}$.

3. Pierre Bourgault est le fils de Jean-Julien. Il ne faut pas confondre avec le politicien indépendantiste.

4. Mais cela dépasse le cadre de cet article.

5. Médard lui-même écrit « Je fais de la sculpture d'art religieux sur bois, taille directe », en réponse à un questionnaire pour la seconde édition de Les Biographies françaises d'Amérique qui sera publiée en 1950.

6. La bibliothèque de Médard inclut des romans, des encyclopédies, des livres sur la technique de menuiserie et de sculpture, des livres d'histoire et d'art, certains datant du XIX ${ }^{\mathrm{e}}$ siècle. Mmv, Entrevue d'André-Médard Bourgault par Jean-François Blanchette, 7 juin 2018, en présence de Benoit Vaillancourt.

7. Nicole Bourgault et Jean Simard, « Saint-Jean-Port-Joli et son patrimoine religieux », www. ameriquefrancaise.org/fr/article-686/Saint-Jean-Port-Joli_et_son_patrimoine_religieux, consulté le 23 juin 2020.

8. Archives de la Côte-du-Sud et du Collège de Sainte-Anne (désormais Acs), F215/1/4, note manuscrite de Jean-Julien Bourgault, « Histoire de la goélette L'Alma », et autre mention d'une deuxième goélette. 


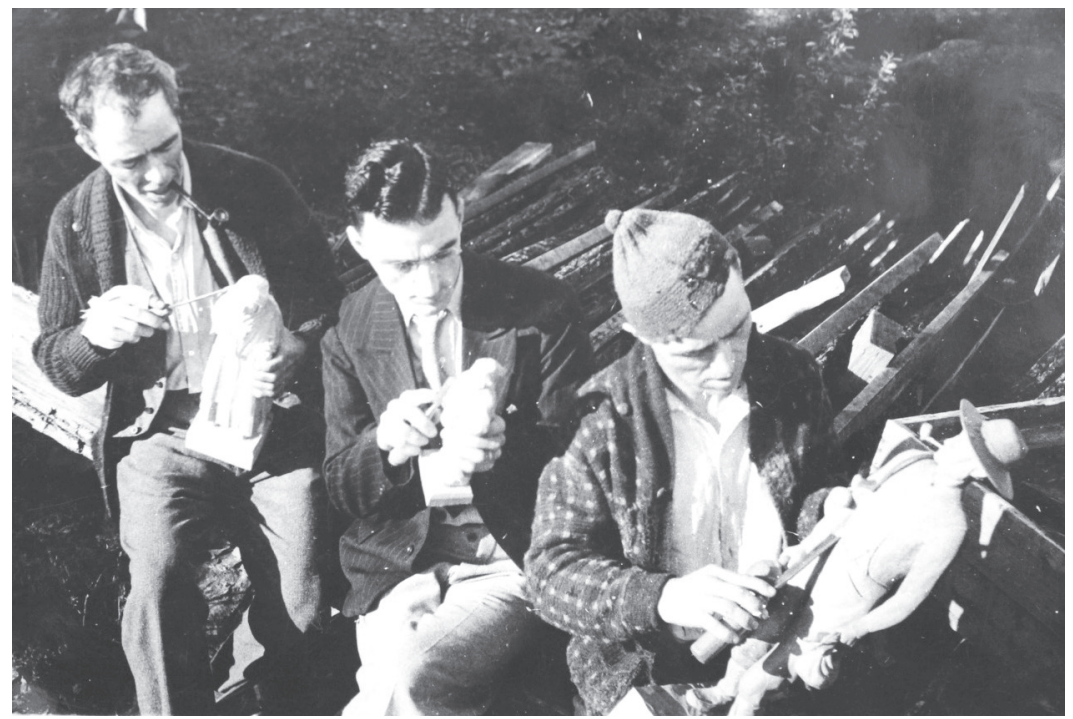

Médard, Jean-Julien et André Bourgault vers 1933

Photo : Acs, Fonds Médard Bourgault, F050/F2,1, nº 17

Émélie, qui vient elle-même d'une lignée de sculpteurs, éveille ses enfants à observer les fins détails des sculptures qu'on y expose. Chez les enfants Bourgault, l'habileté vient sans doute du père, menuisier, mais le goût de l'art et la recherche du beau viennent de leur mère, héritière d'une longue tradition artisanale. C'est ce dont témoigne Médard dans son Journal, en 1940 : «Ma mère était modiste, de descendance des Legros dits SaintLaurent, qui était une famille de ces types ingénieux et adroits de sculpteurs, dessinateurs, orfèvres, menuisiers-charpentiers de navires et marins ${ }^{9}[. .$.$] J'ai$ eu des goûts pour tout ce qui est beau depuis mon enfance, goût qu'avait ma mère qui s'adonnait dans ses loisirs, à confectionner des bouquets artificiels, à des broderies à l'aiguille. Elle était couturière-modiste. Sur les genoux de ma mère, j'appris à prier Dieu, le grand Maître de toutes les beautés naturelles. J'appris à différencier le beau du laid ${ }^{10}$. » Du côté des Legros, le frère d'Émélie, Albert, charpentier de navire, est également un fabricant de maquettes de bateaux ${ }^{11}$, un art prisé dans la communauté ${ }^{12}$. Médard dira

9. Médard Bourgault, Journal, Saint-Jean-Port-Joli, Corporation Maison Musée MédardBourgault, 1991, 114 p. [Miméo, tirage limité], p. 20. Les citations tirées du Journal ont été éditées grammaticalement.

10. Ibid., p. 32 .

11. Acs, F001, D46, Municipalité de Saint-Jean-Port-Joli, Jacques Castonguay, « Jean-Julien Bourgault, artiste sculpteur, Saint-Jean-Port-Joli », [brève biographie] 1978. Angéline Saint-Pierre, André Bourgault, Saint-Jean-Port-Joli, publié à compte d'auteur, 1996, p. 31.

12. Les maquettistes Leclerc deviendront la référence dans ce domaine. 
d'ailleurs que l'influence la plus grande qu'il a eue vient d'Arthur Fournier ${ }^{13}$, un sculpteur au canif qui était ami de son père et qui devint son mentor : « Dans l'hiver de 1921, je me décidai à sculpter un crucifix. Mon vieil ami Arthur Fournier, à qui je dois de m'avoir incité à sculpter le bois, me mit cette idée dans la tête. Il était meublier et s'adonnait beaucoup à la sculpture au couteau. Il sculptait des arabesques. Il sculptait des meubles, des cadres. Il sculptait aussi des épitaphes. Je le visitais très souvent. Il était du même goût que moi ${ }^{14}$. »

\section{Médard Bourgault entre dans l'histoire}

Marius Barbeau, anthropologue au Musée national du Canada, visite Médard Bourgault à l'été $1930^{15}$. Barbeau cherche à documenter la présence de la tradition d'origine française au Québec dans les arts et l'artisanat, et il en fait la promotion. Il est attiré par les lions qui décorent la lucarne de la maison de Médard qu'il visite ${ }^{16}$. Il est épaté de constater la riche production de cet artisan qu'il relie aussitôt à la tradition des grands artistes issus de la Nouvelle-France. Il achète des œuvres de Médard et il le fait connaître dans les réseaux de collectionneurs, les milieux culturel, religieux, politique et médiatique au Canada et à l'étranger. Des articles dithyrambiques, comme celui du juge Camille Pouliot dans La Presse en 1932 $2^{17}$, contribuent à le faire connaître et à présenter son grand talent à l'élite québécoise, canadienne et états-unienne. Des commandes arrivent de nombreux décideurs, car le prestige d'un poste exige qu'on y mette un Bourgault à l'honneur sur son bureau ou sur un mur de l'organisation ou de l'entreprise ${ }^{18}$.

Rappelons brièvement le contexte. Depuis les années 1930, le gouvernement du Québec désire remettre à l'honneur les métiers artisanaux et utiliser ce savoir-faire dans la promotion du tourisme qui est le secteur économique le plus rentable après les mines et la pâte à papier $^{19}$. Le développement d'un réseau routier à travers la province, dont l'inauguration de la route de la Gaspésie en 1928, et l'amélioration des services d'hébergement attirent les touristes qui sont nombreux à passer par

13. J.-Arthur Fournier, Mémorial de Saint-Jean-Port-Joli, Musée de la mémoire vivante, [1923] 2012, XXI-556 p.

14. Médard Bourgault, Journal, op. cit. p. 33.

15. Médard écrit dans son Journal, rédigé en 1940, que la rencontre a eu lieu en 1929 : Médard Bourgault, Journal, op. cit., p. 35. Mais Marius Barbeau passe tout l'été 1929 en Colombie-Britannique. Par contre, il écrit, dans une note de terrain en 1930, qu'il a rencontré Médard : Musée canadien de l'histoire (désormais Мсн) Fonds Marius Barbeau, Boîte 284, F1D/Dossier 55 : Bourgault.

16. Médard Bourgault, Journal, op. cit., p. 35.

17. La Presse, 27 janvier 1932.

18. La correspondance du Fonds Médard Bourgault (Acs, F050) est éloquente à cet égard.

19. Nicole Neatby, From old Quebec to La Belle Province. Tourism Promotion, Travel Writing, and National Identities, 1920-1967, Montréal, McGill Queen's University Press, 2019, XvI-344 p. 
Saint-Jean-Port-Joli, quand ce n'est pas en soi la destination ${ }^{20}$. Les touristes peuvent désormais se rendre dans les lieux de villégiature en automobile et non plus seulement en bateau ou en train. Comme la route passe devant sa maison, Médard installe une petite table à l'extérieur pour vendre ses pièces aux touristes. Mais dans le milieu, pourtant familier avec la venue de touristes qui achètent des textiles des Chamard et des maquettes de bateaux des Leclerc, on ne voit pas bien la vente de petits objets pour en tirer un profit parce que, dans la société traditionnelle, on a l'habitude d'offrir ce genre d'objet en cadeau à des êtres chers ${ }^{21}$.

Médard ne peut subvenir à la tâche. Dès 1931, il demande l'aide de ses frères Jean-Julien et André pour l'assister. Leurs neveux, Léon et Alphonse Toussaint, de même qu'une jeune sœur de Médard, Yvonne, se joignent à eux pour produire des figurines pour le marché touristique ${ }^{22}$ pendant que Médard se consacre aux œuvres majeures, comme il l'indique lui-même à Marius Barbeau : «Nous avons beaucoup de travail ; l'on ne peut pas fournir à toutes les demandes. Pour moi, je travaille presque toujours dans les grosses pièces. C'est un surplus pour nous. Nous vous devons cette prospéritéé ${ }^{23}$.

Les journaux louangent cette production québécoise qui raconte la vie traditionnelle du Québec. Damase Potvin écrit dans un article du 13 juillet 1931 : «On cite tout particulièrement, parmi ses scènes champêtres [de Médard], une scène d'arrachage de souches qui serait tout simplement merveilleuse de réalisme, de naturel, de vie, de proportion juste, de lignes harmonieuses ${ }^{24}$. » En réponse au docteur Gabriel Nadeau, un client important de Rutland au Massachusetts, Médard écrit : « Les pièces les plus populaires sont les types d'habitants canadiens-français et les scènes ou bas-reliefs de la vie des champs : les bûcherons, les pêcheurs gaspésiens ; les chiens, les attelages de bœufs et les chevaux sont aussi assez populaires. Mais la plus populaire de toutes est le type d'habitant ou habitante tel que fileuse et tricoteuse ou autres. Les sujets religieux sont assez demandés aussi. » Il indique que ses clients viennent de partout et il précise : «Nous vendons beaucoup aux Canadiens français, surtout dans les pièces dispendieuses ${ }^{25}$. »

20. Jean-Marie Gauvreau croyait que l'Auberge du Faubourg, qui se présente comme «L'auberge de l'artisanat » était la meilleure réussite de la mise en valeur du patrimoine artisanal local (Jean-Marie Gauvreau, «Le Problème de l'artisanat dans la Province de Québec », Étude présentée au ministère de l'Industrie et du commerce, avril 1942, BANQ, Fonds Jean-Marie Gauvreau, MSS2/2/6, 152 p.

21. Мсн, Fonds Jean-François Blanchette, témoignage de Pierre Bourgault lors d'une rencontre du Groupe de travail en art populaire avec des artistes de Saint-Jean-Port-Joli, 13 juin 2007.

22. Gérard Ouellet, Ma paroisse, Saint-Jean Port-Joly, Québec, Éditions des Piliers, 1946, p. 301.

23. Acs, F050/1/4, Médard Bourgault à Marius Barbeau, 28 décembre 1934.

24. Cité par Jean-Marie Gauvreau, BANQ, MSS2/1/22, «L'artisanat dans la Province de Québec », conférence du 12 février 1937, p. 25-27.

25. Acs, F050/1/4, Correspondance, 19 décembre 1935. 
L'analyse de la correspondance de Médard éclaire son parcours et sa démarche artistique. Elle nous révèle son style qui évolue à la suite des suggestions, des critiques et des commandes nombreuses qu'il reçoit. Par exemple, Marius Barbeau le conseille sur ses Christs trop souffrants ${ }^{26}$ et lui suggère de ne pas aller aux beaux-arts ${ }^{27}$. Georges Bouchard, auteur de Vieilles choses, vieilles gens (1926), lui recommande de continuer à produire de l'art paysan, ce qui a fait sa réussite ${ }^{28}$. Jean-Marie Gauvreau insiste pour qu'on n'utilise plus la polychromie qui ne permet pas de distinguer les sculptures des produits " en affreux plâtre peinturluré29 », qu'on trouve en quantité sur les marchés. Selon toute apparence, c'est aussi Jean-Marie Gauvreau, qui a été formé à l'ébénisterie classique à l'école Boulle de Paris ${ }^{30}$, qui suggère à Médard de suivre des cours d'appoint aux beaux-arts ${ }^{31}$ ou qu'un conseiller vienne de France travailler avec lui et son frère Jean-Julien ${ }^{32}$. Mais Médard ne se laisse pas tenter par les invitations à suivre les maitres classiques. De plus, comme il a déjà sept enfants (trois garçons et quatre filles) en $1935^{33}$, il doit maintenir le rythme de la production pour subvenir aux besoins de la famille. Il n'est donc pas question de quitter Saint-Jean-Port-Joli. Ils sont d'ailleurs assez occupés avec les commandes et leurs apprentis qui sont surtout locaux bien que certains stagiaires viennent de l'extérieur. Ainsi, Lucienne Boyer de Montréal y fait quelques séjours entre 1934 et 1937, sans doute à la suggestion de Paul Gouin puisqu'elle sculpte pour lui, à partir d'un dessin de l'artiste Maurice Raymond, un magnifique jeu d'échecs commémoratif de la bataille des Plaines d'Abraham ainsi qu'un impressionnant coffret ${ }^{34}$.

26. Acs, F050/1/4, Correspondance, Marius Barbeau à Médard Bourgault, 11 avril 1935.

27. Acs, F050/1/2, Correspondance, Marius Barbeau à Médard Bourgault, 2 décembre 1932.

28. Acs, F050/1/2, Correspondance, Georges Bouchard à Médard Bourgault, 12 décembre 1932.

29. Jean-Marie Gauvreau, Artisans du Québec, Montréal, Éditions du Bien public, 1940, p. 123.

30. « Durant l'entre-deux-guerres, l'école [Boulle] devient un des soutiens de l'art déco, mouvement auquel elle donne de nombreux décorateurs. » fr.wikipedia.org/wiki/École_Boulle, consulté le 21 juin 2020.

31. Je présume que c'est Gauvreau qui a proposé à Médard d'aller suivre des cours à l'École des beaux-arts sous la supervision de Charles Maillard qui est directeur général des beaux-arts pour la province. Voir Acs, F050/1/2, Correspondance, Marius Barbeau à Médard Bourgault, 2 décembre 1932. Voir aussi fr.wikipedia.org/wiki/Charles_Maillard_(1887-1973), consulté le 23 juin 2020.

32. Gauvreau a insisté pour que des professeurs viennent de France conseiller les ateliers qu'il a aidés. Voir Jean-François Blanchette, « Marius Barbeau et l'authenticité de la tradition en art populaire », Rabaska, vol. 13, 2015, p. 141-151.

33. Acs, F050/1/4, Correspondance, 19 décembre 1935.

34. Cet ensemble exceptionnel fut le coup de cœur du conservateur Christian Denis dans l'exposition Sortir de ma réserve tenue au Musée de la civilisation en 2018-2019 (communication personnelle et correspondance, avril 2018). Voir collections.mcq.org/objets/99840, consulté le 23 juin 2020. Voir aussi Acs, F050/1/5, Correspondance de Lucienne Boyer adressée à " Chers professeurs », 4 septembre 1937. 
Très tôt, Médard passe de l'art paysan à l'art religieux, car cela lui permet d'éviter les pièges du marché touristique et de se consacrer à la création d'œuvres originales qui correspondent plus à ses goûts personnels. Parmi ses premières réalisations majeures dans ce domaine, il faut mentionner la statuaire et le mobilier de l'église Sainte-Anastasie de Lachute construite sous la direction de l'architecte Eugène Perron et qui occupe le sculpteur en 1936 et $1937^{35}$. Comme Médard n'a pas de catalogue, ne désire pas se répéter et qu'il veut faire œuvre nouvelle, le projet est élaboré par l'architecte, l'artiste et les représentants de la paroisse qui s'échangent correspondance, dessins et échantillons au cours du processus de création.

\section{" L'École de sculpture sur bois Médard \& Jean-J. Bourgault", 1940-1942}

La crise économique qui débute en 1929 amène des dirigeants politiques et divers intervenants à développer des emplois et des marchés pour les mains habiles. Jean-Marie Gauvreau (directeur de l'École du meuble), Albert Tessier (photographe, cinéaste, professeur et défenseur de la langue française, auteur déjà de nombreux films), Paul Gouin (collectionneur et conseiller culturel du Gouvernement), Georges Bouchard (député de Kamouraska à la Chambre des communes, cofondateur des Cercles de fermières), et le lieutenant-colonel Wilfrid Bovey (de la Canadian Handicraft Guild de Montréal) s'impliquent dans la recherche et la promotion d'artisans qui perpétuent les traditions et qui vont nourrir la vision officielle d'améliorer le tourisme en favorisant et en mettant en valeur la riche tradition artisanale ${ }^{36}$. Esdras Minville de l'École des hautes études commerciales, responsable de l'inventaire des ressources naturelles pour le gouvernement du Québec, accepte la proposition de Jean-Marie Gauvreau, de former des équipes, en $1938^{37}$, pour faire l'inventaire de l'artisanat. On demande par ailleurs aux enquêteurs de décrire également l'organisation du tourisme et de faire des recommandations pour son amélioration. Albert Tessier et Paul-Émile Borduas sont de la partie. Grâce aux résultats de ces enquêtes et à la suite de nombreuses démarches, Jean-Marie Gauvreau réussit à convaincre le gouvernement d'investir dans la création d'ateliers de formation et de production artisanale dans les régions rurales du Québec. Des artisans vont recevoir le financement nécessaire grâce au programme fédéral-provincial

35. Acs, F050/1/5, Correspondance pour les années 1936 et 1937. À lui seul, ce projet mériterait une étude des échanges entre les divers intervenants.

36. Voir «L'inventaire des ressources artisanales » dans Jean-François Blanchette, Du coq à l'âme, l'art populaire au Québec, Gatineau, Musée canadien de l'histoire, «Collection Mercure, Études culturelles » 85, 2014, p. 72-89. Voir aussi Nicole Neatby, op. cit.

37. Les inventaires des ressources naturelles commencent en 1937. Cette année-là, seule l'équipe de Gauvreau inclut l'artisanat dans ses observations. 
Bilodeau-Rogers d'aide à la jeunesse ${ }^{38}$. Gauvreau est convaincu qu'il y a une demande locale autant que touristique pour les produits que ces ateliersécoles vont développer, car on trouve sur les marchés québécois une immense variété d'articles importés d'Europe, d'Asie et d'ailleurs. Ces importations diminuent pendant la guerre de 1939-1945, ce qui favorise l'artisanat local et l'industrie canadienne, aux yeux de Gauvreau ${ }^{39}$.

Les démarches de ce dernier mènent à la création d'un " atelierécole $»^{40}$ de sculpture sur bois à Saint-Jean-Port-Joli en 1940, malgré les objections des gens des beaux-arts qui y voient une concurrence, au dire même de Gauvreau ${ }^{41}$. Une entente est signée entre le ministère des Affaires municipales, de l'industrie et du commerce et Médard et Jean-Julien Bourgault ${ }^{42}$ pour la location d'un atelier « complètement outillé et équipé » pour former simultanément quinze élèves jusqu'au 31 mars 1941 au coût de trente dollars par semaine. L'atelier que Médard partage avec son frère Jean-Julien est donc rapidement agrandi pour subvenir à ces besoins. Une allocation d'un dollar par apprenti permet l'achat des petits outils et le bois pour leurs travaux. Par ailleurs, l'un des deux « instructeurs », Médard ou Jean-Julien, reçoit soixante dollars par semaine pour la formation pendant cette brève période. Ils s'engagent de plus à se conformer aux instructions de Jean-Marie Gauvreau « au point de vue initiation des élèves selon les règles de l'art. » Ce dernier donnera d'ailleurs chaque mois « quelques notions de technologie du bois ${ }^{43}$. »

Les jeunes, choisis par des membres influents de la communauté dans les diverses régions du Québec, arrivent pour le début des cours le 5 décembre à l'École de sculpture sur bois Médard \& Jean-J. Bourgault ${ }^{44}$. À l'automne 1940, les quinze élèves sont Denis Bernier de Saint-Jean-Port-Joli, Stanislas Boudreau de La Tuque, André Dubé de Saint-Roch-des-Aulnaies, Jules-Gonzagues Bois de Sainte-Anne-de-la-Pocatière, Stanley Bouchard

38. Voir Christian Blais, "Introduction historique, $20^{\text {e }}$ législature, $3^{\text {e }}$ session, " Travaux parlementaires pour 1937 à www.assnat.qc.ca/fr/travaux-parlementaires/assemblee-nationale/20-3/ introduction-historique.html?retourVersHistoire $=$ oui, consulté le 23 juin 2020. Au sujet d'écoles d'artisanat qui recevront de l'aide, voir Jean-François Blanchette, « Marius Barbeau et l'authenticité de la tradition en art populaire », op.cit.

39. Jean-Marie Gauvreau, « Le Problème de l'artisanat ... », op. cit., p. 138. Jean-Marie Gauvreau (1903-1970) semble avoir apporté son soutien aux frères Bourgault tout au long de leurs carrières.

40. C'est le terme utilisé par Albert Lévesque du bureau d'Initiation fédérale-provinciale de la jeunesse chômeuse au ministère des Affaire municipales, de l'industrie et du commerce du Québec, qui écrit à Jean-Julien et Médard Bourgault le 15 novembre 1940 : Acs, F215/9/1.

41. Jean-Marie Gauvreau, « Le Problème de l'artisanat ... », op. cit., p. 75.

42. Acs, F215/9/1 « Mémoire de convention ». L'année 1940 est indiquée sans préciser la date exacte.

43. Jean-Marie Gauvreau, « Le Problème de l'artisanat ... », op. cit., p. 75.

44. C'est le nom qui est imprimé comme en-tête de lettre. Acs, F050/1/8, lettre de Médard Bourgault au père F. Prud'homme datée du 4 décembre 1940 où on peut lire « C'est l'ouverture de notre école de sculpture demain. » 
de Baie-Saint-Paul, Paul-André Caron de Saint-Jean-Port-Joli, Paul-Émile Caron de Saint-Roch-des-Aulnaies ${ }^{45}$, Gilbert Gamache de Saint-Georgesde-Beauce, Édouard Lafrenière de Saint-Justin de Maskinongé, Rosario Marcoux de Saint-Apollinaire, Robert Matton de Trois-Rivières, Trefflé Picard de Saint-Roch-des-Aulnaies, Richard Richard de Sainte-Anne-dela-Pocatière, Roland Richard de Sainte-Thérèse de Gatineau et Wilfrid Richard de Rivière-Ouelle ${ }^{46}$. À la session d'hiver 1940, René Lavoie de Baie-Saint-Paul se joint au groupe ${ }^{47}$.

Le programme d'Aide à la jeunesse procure une aide financière aux élèves pour la durée seulement de l'entente. La production des élèves, qui doit avoir une "valeur commerciale », peut être vendue et les revenus retournés au ministère moins une commission de $25 \%$. Enfin, Jean-Julien et Médard s'engagent à garder les élèves à leur compte à la suite de cette formation de six mois, le but de l'aide financière visant strictement à donner un coup de pouce au démarrage de l'entreprise. Voilà pourquoi il s'agit d'un atelier de formation plutôt qu'une école proprement dite. Cela oblige les deux maîtres sculpteurs à tenir à bout de bras le développement de cette initiative et la rendre rentable.

L'apprentissage avec interaction pratique entre maîtres et apprentis est la base de cet atelier. On apprend en observant les maîtres et en développant son habileté. Des cours de dessin sont donnés, sans doute par Jean-Julien qui excelle en ce domaine. Plus d'une centaine de dessins ont été conservés de cette première année par le fils de Médard, André-Médard. Ils sont signés et datés. Leur examen nous permet de découvrir les thèmes traités comme les arts décoratifs, la vie religieuse, la vie quotidienne, les personnages influents de "l'école », l'art animalier. Les dessins, pour la plupart peu élaborés, représentent des visages d'homme et de femme, de face et de profil, des personnages religieux comme le Christ, des saints, des moines, des scènes de vie traditionnelle. Sans doute que certains de ces thèmes sont inspirés de modèles pris sur le vif ou de sujets de la vie traditionnelle. Mais l'analyse de ces dessins nous indique que des figurines italiennes représentant des paysans ou des religieux sont également utilisées comme modèles ${ }^{48}$. Cet

45. Né à Saint-Roch-des-Aulnaies, Paul-Émile Caron s'installera à Saint-Jean-Port-Joli quand il quittera l'atelier de Médard et Jean-Julien.

46. L'Action catholique, 20 décembre 1940.

47. L'Action catholique, 16 mai 1941.

48. C'est ce que démontre la comparaison avec les sculptures italiennes de la maison ANRI publiées dans Philly Rains et Donald Bull, ANRI Woodcarvings : Bottle Stoppers, Corkscrews, Nutcrackers, Toothpick Holders, Smoking Accessories, and More, Arglen (Pennsylvanie), Schiffer Publishing Ltd, 2001, 272 p. Des modèles importés d'Europe servaient également de modèles à l'atelier de poterie de Beauceville, selon Daniel Cogné, Richard Dubé et Paul Trépanier, Céramique de Beauce, Québec, Éditions Gid, 2004, p. 56. Cette utilisation de modèles étrangers est également courante dans les beaux-arts (John R. Porter et Jean Bélisle, La Sculpture ancienne au Québec, Montréal, Éditions de 
atelier-école institutionnalise une forme d'apprentissage issue de la tradition populaire que les Trois Bérets ont pratiquée depuis leurs premiers ateliers - l'interaction avec les élèves - ainsi que la technique de la taille directe.

La durée de l'entente, qui à l'origine devait se terminer le 31 mars, est modifiée en raison du retard à la signer et du fait que l'agrandissement de l'atelier n'a pu être achevé avant le début du mois de décembre. Comme ce genre de soutien financier dure habituellement 26 semaines, l'entente est prolongée jusqu'au 3 juin. La synthèse du budget de ces six mois indique qu'en tout et partout, salaires et dépenses inclus, on en a retiré un revenu net de 467,92 \$. La production des seize élèves en six mois, si on la considère séparément, a totalisé une valeur, en sculptures vendables, de seulement 528,40 \$. Cela démontre bien qu'il faut du temps pour former un bon sculpteur et qu'on ne peut pas tirer profit de la production avant un certain temps. Le gouvernement ne renouvelle pas l'entente de location de l'atelier de Jean-Julien et de Médard après le 3 juin. De plus, les apprentis ne reçoivent plus les allocations d'aide à la jeunesse, la priorité du programme portant dorénavant sur les centres de formation qui contribuent à l'effort de guerre ${ }^{49}$. Les apprentis de Médard et Jean-Julien sont devenus leurs employés et les ventes doivent permettre de leur verser un salaire. On ne sait rien à ce sujet, sinon qu'à l'automne 1941, la Photo Gelatine Engraving d'Ottawa commande pour 7000 \$ de sculptures aux Bourgault, « ce qui assurait l'avenir de l'atelier pour un $a^{50}$. » Médard écrit à Albert Tessier le 10 février 1942. L'en-tête de sa lettre se lit toujours « École de sculpture sur bois Médard \& Jean-J. Bourgault » : « Ça va très bien avec nos élèves. Ils font du bon travail à présent, mais la guerre va peut-être intervenir [nuire] dans nos espérances. Nous donnons des cours le soir à nos familles cet hiver. C'est malheureux que le gouvernement ait abandonné, car avec de l'aide nous aurions pu créer un bon centre d'art ici. C'était là nos espérances. Nos élèves ne parlent plus de nous quitter, personne, car ils sont bien encouragés. Nous leur payons un très bon salaire. Nous en avons trois de mariés ; un seul nous a laissés, non pas parce que ça ne faisait pas son affaire. Il s'est marié et il avait sa maison à Saint-Roch-des-Aulnaies. Mais il travaille pour nous quand même. Nous les payons toutes les semaines. Ils se font des salaires, quelques-uns de 20-26 et 30 piastres par semaine ${ }^{51}$. " Par contre, au cours de la même année, Médard et Jean-Julien ferment leur école et Médard inscrit dans le cahier où il tient son budget, "Nous n'avons plus d'élèves ${ }^{52}$. » Comment expliquer ce revirement ? Quel est l'état du marché 1'homme, 1986, p. 275-278.

49. Jean-Marie Gauvreau, « Le Problème de l'artisanat ... », op. cit., p. 76.

50. Ibid., p. 77.

51. Acs, F050/1/10, Correspondance, 1942.

52. Acs, F050/4/3, Cahier « Travail de $1950 »$ avec des notes datées, antérieures à 1950. 
pour les sculptures produites à l'atelier? Médard et Jean-Julien pouvaient-ils mener simultanément un atelier de formation tout en se gardant le temps nécessaire pour créer?

De la cuvée de 1940-1941, certains élèves font carrière en sculpture. Paul-Émile Caron est ingénieux et il a du talent. Il désire innover et se démarquer de ses maîtres en produisant des sculptures pour les ouvriers d'usine qui ne peuvent se payer des œuvres d'art comme celles produites par Médard et Jean-Julien. Il forme de nombreux apprentis qui travaillent parfois à l'atelier, mais aussi parfois chez eux, ce qui permet aux femmes d'avoir un revenu tout en s'occupant de leur famille ${ }^{53}$. André Dubé retourne à Saint-Roch-des-Aulnaies et fait carrière comme animalier. Il forme son frère Clément qui aura ensuite son propre atelier. Leur frère Arthur, autodidacte, formera son fils Denis qui sculpte toujours ${ }^{54}$. Stanley Bouchard et René Lavoie de Charlevoix retournent dans leur région et font carrière. Stanislas Boudreault, Trefflé Picard, Richard Richard et Wilfrid Richard continuent dans cette voie, mais les autres font d'autres métiers ${ }^{55}$.

\section{Projet d'un nouvel atelier-école en 1953}

À partir de 1944, le programme d'Aide à la jeunesse contribue de nouveau à la formation d'artisans non rattachés aux métiers liés aux efforts de guerre. On verra plus loin que les apprentis d'André Bourgault y auront accès. Il devait y avoir une grande demande pour ce type d'apprentissage, car Médard discute la possibilité de rouvrir un atelier-école en 1953. Il reçoit alors des propositions concrètes qui prévoient des salaires pour le professeur et pour les apprentis. Par contre, l'atelier-école doit payer une partie du salaire de ces derniers de façon croissante chaque semaine ${ }^{56}$, car on croit qu'ils pourront produire pour le marché et que ces revenus compenseront ces frais. Jean-Marie Gauvreau, qui est alors président de l'Office provincial de l'artisanat et de la petite industrie - une agence du ministère de l'Industrie et du commerce participe aux négociations ${ }^{57}$ de même que Fernand Lizotte, alors député de l'Islet à l'Assemblée législative du Québec ${ }^{58}$ en essayant de démontrer aux fonctionnaires que les apprentis ne pourront pas être productifs avant plusieurs mois. Pour aider Médard dans le financement de l'entreprise, le ministère offre

53. L'entreprise passera aux mains de son fils Jocelyn en 1966 et c'est un tout nouveau pan de l'histoire qui s'ouvrira. Mmv, Entrevue de Jocelyn Caron par Jean-François Blanchette, 3 juin 2016.

54. Mмv, Entrevue de Denis Dubé par Jean-François Blanchette, 30 septembre 2016.

55. Jacques Trépanier, La Patrie du dimanche, $1^{\text {er }}$ novembre 1959, p. 116.

56. Acs, F050/1/21, Correspondance entre Louis-Philippe Audet, directeur pédagogique, chef de la Culture populaire, Service de l'Aide à la jeunesse, ministère du Bien-Être social et de la jeunesse à Médard Bourgault, les 12 et 13 mars 1953.

57. Acs, F050/1/21 Jean-Marie Gauvreau, Président, Office provincial de l'artisanat et de la petite industrie, ministère de l'Industrie et du commerce, à Médard Bourgault, 27 avril 1953.

58. Acs, F050/1/21 Médard Bourgault à $\mathrm{D}^{\mathrm{r}}$ Fernand Lizotte, 20 avril et 7 mai 1953. 
même de payer un loyer de 75 \$ par mois, mais Médard décide finalement de ne pas y donner suite. Sans doute trouve-t-il que son salaire est négligeable et que les exigences du gouvernement sont irréalistes.

\section{Commandes en art religieux}

Médard et Jean-Julien partagent le même atelier jusqu'à ce que Jean-Julien fonde son atelier de mobilier religieux en 1949 afin de satisfaire une demande croissante et apparemment intarissable. La commande de l'École ménagère régionale des Sœurs de Sainte-Anne de Saint-Jacques-del'Achigan (Montcalm), entre 1948 et 1951, est un bel exemple de projet qui concerne autant Médard que Jean-Julien ${ }^{59}$. Sœur Marie-Jeanne-de-France, la directrice, demande à Médard un chemin de croix en haut-relief d'environ 18 po x 16 po : «Vous pouvez le faire découpé, et lui donner le plus de relief possible, pour qu'on le voie de loin, et qu'il soit très beau, n'est-ce pas $?^{60} \gg$. La communauté commande également des statues en ronde bosse et une crèche de Noël en plus de pièces d'ameublement que produit Jean-Julien ${ }^{61}$ : « Nous avons reçu les modèles de votre frère pour la table de communion; je vais en faire part à l'architecte pour que tout marche avec l'ensemble et le plus tôt possible vous recevrez des nouvelles. Ce qui est certain, c'est que nous lui donnerons l'entreprise ; quant aux motifs, nous en faisons l'étude actuellement $[\ldots]^{62}$ » Elle ajoute : « Je serai si fière de dire que toute la décoration est l'œuvre des sculpteurs "Bourgault". Je pense que nous aurons une très belle chapelle [...]. C'est là qu'auront lieu nos retraites et tous nos Congrès. Je tiens à ce que l'artisanat soit à l'honneur ${ }^{63}$. » Ce projet remarquable attire l'intérêt des architectes et connaisseurs de l'époque et contribue à la réputation déjà grande de Médard et Jean-Julien ${ }^{64}$.

Descommandes viennent également des États-Unis. Eugène R. Gallagher, de la paroisse Saint-Paul Apôtre d'Irvington au New-Jersey commande un chemin de croix de Médard. Il précise ce qu'il désire, le 8 mai 1950 : «J'aimerais avoir ces stations sculptées dans un style moderne modifié, sans aller à l'extrême ni au grotesque. Je les voudrais sculptées dans le style de la tête du Christ achetée chez vous l'an dernier ${ }^{65}$. » Il indique à Médard qu'il

59. Le 20 septembre 1949, l'École ménagère de Saint-Jacques emménage dans un nouveau bâtiment sous le nom d'École supérieure d'enseignement ménager, aujourd'hui le Collège EstherBlondin : www.collegeblondin.qc.ca/le-college/historique/ consulté le 3 juin 2020.

60. Acs, F050/1/16, Sœur Marie-Jeanne-de-France, directrice, École ménagère régionale des Sœurs de Sainte-Anne, Saint-Jacques (Montcalm), à Médard Bourgault, le 15 novembre 1948.

61. Acs, F050, Correspondance entre le 9 novembre 1948 et le 15 juillet 1951.

62. Acs, F050/1/16, Sœur Marie-Jeanne-de-France, le 25 décembre 1948.

63. Acs, F050/1/17, Sœur Marie-Jeanne-de-France, le 2 février 1949.

64. En plus d'être, en 2020, un modèle d'aménagement d'un trésor patrimonial grâce au programme novateur Zone 3D qu'on y a installé avec goût en 2017, la chapelle est présentée en 3D sur le site visites360.collegeblondin.qc.ca/fablab.html, consulté le 3 juin 2020.

65. Acs, F050/1/18, Correspondance, 1950. 
discute avec Jean-Julien la production des fonts baptismaux. Le chemin de croix est expédié en octobre 1950. Il a coûté $1400 \${ }^{66}$, ce qui indique qu'il devait être exceptionnel par sa dimension et sans doute en haut-relief.

\section{Un art canadien-français}

À divers endroits de son Journal, Médard plaide pour un art religieux canadien-français ${ }^{67}$ qu'il oppose à l'art moderne qui est en vogue dans le milieu des arts. Voici en bref son argument : «Quand je contemple les œuvres de nos aïeux, dans le petit nombre qu'il nous reste de nos vieilles églises, je me dis que je ne suis rien à comparer à ces artistes qui étaient de leurs temps. Mais si nous sommes dans un temps nouveau, efforçons-nous de créer chez nous ce qui est nouveau, non pas ce qui est étranger. Nous aurions tant à créer au sujet de notre race, en fait de martyrs et de croyances religieuses. Pourquoi nos Christs ne seraient-ils pas d'expression canadienne ? Pourquoi ne pas avoir nous aussi nos vierges canadiennes comme en France, telles Notre Dame des Canadiens français ou de Québec, Notre-Dame aux blés, Notre-Dame de l'agriculture, Notre-Dame du Saint Laurent, Notre-Dame du Canada ? Enfin, il y aurait beaucoup à créer. Il s'agit un peu d'imagination ${ }^{68}$. [...] Il faut toujours que ça soit d'après des créations d'artistes étrangers. On m'arrive avec une petite image ou statuette, et on me demande toujours la même chose : "Pouvez-vous me faire quelque chose pour approcher cela autant que possible ?"69 [...] Il faut gagner son pain quotidien, il faut accepter si l'on veut manger un peu. Quand il faut avoir du pain sur la table pour dix-huit bouches, il faut accepter des choses avec regret ${ }^{70}$. » Il ajoute avec malice : "Mais par contre, je les triche un peu. Je fais à mon idée dans les expressions, des expressions canadiennes qui sont dans mon entourage autant que possible et dans le drapé. J'ai mon style. Mes clients en sont satisfaits, sans s'apercevoir, la plupart du temps, que je me suis fiché de leur modèle ${ }^{71}$. »

Médard s'oppose au style moderne qui simplifie à outrance le sujet pour ne laisser que l'idée du sujet : « Souvent, on m'arrive pour faire sculpter une statue de la Sainte Vierge. Il ne faut pas de buste, il faut un buste plat. Il ne faut pas que la moindre forme des cuisses paraisse dans le drapé. Dans une douille quoi ! C'est ce à quoi ça ressemble. La Sainte Vierge pourtant était une femme faite comme une femme, comme nos mères canadiennes, pas autre

66. Acs, F050/4/3 « Travail de $1950 »$, octobre.

67. Gérard Ouellet fait la promotion des idées de Médard dans L'Action catholique du 12 février 1939. Voir aussi la correspondance de Marc Couillard-Després, prêtre vicaire de Saint-Jean Port-Joli, à Gérard Ouellet, 16 janvier 1938 (Acs, F050/1/6).

68. Médard Bourgault, Journal, 1991, op. cit., p. 46.

69. Médard Bourgault, Journal, 1991, op. cit., p. 100.

70. Loc. cit.

71. Loc. cit. 
chose $^{72}$. $\gg$ Les paroisses qui désirent des œuvres bien identifiées au style de Médard Bourgault sont nombreuses. La demande du curé G. E. Desfossés, de la paroisse Sainte-Anne de Val-Côté, est explicite : "Tel qu'entendu, les statues présentent des figures clairement distinctes, rien de soi-disant "moderne"; nos gens veulent bien encore "reconnaître ceux qu'ils prient" ${ }^{73}$."

Parmi les œuvres originales créées par Médard, il y a de nombreuses représentations de la Vierge Marie, Notre-Dame des blés, Notre-Dame des poètes, Notre-Dame des flots, qui est vendue au Musée du Québec ${ }^{74}$ et NotreDame des habitants portant une gerbe de blé, acquise par l'abbé Tessier qui encourage Médard dans cette voie de créativité originale ${ }^{75}$. Cette dernière statue est d'ailleurs choisie par Marius Barbeau pour figurer dans le livre des plus grandes représentations de la Vierge au monde, The World's Great Madonas $^{76}$.

\section{L'art profane}

Il est possible que la baisse lente de l'intérêt pour les sculptures religieuses au milieu des années 1950 et le désir de créer un art typiquement canadienfrançais conduisent Médard à s'orienter vers la production d'œuvres inspirées de la nature. Le bois d'épave ou échoueries se retrouve en grande quantité sur les rives du fleuve. Les apparences naturelles de ces bois le mènent vers une autre forme de créativité. André-Médard raconte : «Quand mon père a acheté le terrain au bord de la mer, il a commencé à faire des nus, il a commencé à décorer son terrain, mais avec des bois de grève. Il prenait des bois de grève, puis le morceau de bois l'inspirait pour telle œuvre, parce qu'il s'intéressait beaucoup à la mythologie grecque. Il avait sculpté les Trois Grâces. Mais au lieu des cygnes, il nous a dit : "Moi j'ai fait des outardes, parce qu'ici, on n'a pas ça, des cygnes." Alors c'est trois femmes avec des outardes entre les jambes [...]. Il avait sculpté la coureuse des grèves [...]. Et après ça, il avait sculpté des pièces comme ça sur le terrain, comme Le Dieu des vents et des tempêtes. [...] Puis, il y a beaucoup d'œuvres qu'il a créées comme ça, qui avaient rapport à la mer, au fleuve et aux montagnes de notre région : Le Chant de la mer, Les Amours de Laurent, Les Laurentides. Mon père disait: "En Europe, eux autres, ils ont beaucoup de légendes. Pourquoi nous, on ne composerait pas avec ce qu'on a autour de nous autres ?" Puis à un moment

72. Loc. cit.

73. Acs, F050/2/2, Correspondance, 20 mars 1962.

74. Acs, F050/1/10, Correspondance, Paul Rainville, conservateur au Musée du Québec à Médard Bourgault, le 10 septembre 1942.

75. Acs, F050/1/8, Albert Tessier à Médard Bourgault, le 30 décembre 1940.

76. La photo de Notre-Dame des habitants et le texte de Marius Barbeau sont publiés dans Cynthia Pearl Maus, The world's great Madonnas : an anthology of pictures, poetry, music, and stories centering in the life of the Madonna and her Son, New York, Harper and Brothers, 1947, p. 535-537. 
donné, il avait fait Enlèvement de Laurentides par Appalaches ${ }^{77}$. »

Les cahiers de vente de Médard indiquent que de nombreux clients, individus comme compagnies, acquièrent ce genre de pièces dès $1955^{78}$. Une admiratrice écrit à Médard le 24 septembre 1957 : « Ayant vu et admiré la magnifique plaquette Le Chant de la mer que notre chauffeur français a choisie lors de notre passage la semaine dernière à votre atelier, je brûle d'envie d'en posséder une ${ }^{79}$. » Pour sa part, le père Antonin Pelletier du monastère des Dominicains à Québec écrit à Médard le 15 juillet 1959 : «Auriez-vous la bonté de me sculpter "Le Chant de la Montagne" et le "Chant de la Mer" aux mêmes dimensions que celles que j'ai achetées chez-vous l'an dernier [...] et de manière à ce que le jeune homme et la jeune fille se fassent face $?^{80}$ »

André-Médard raconte : «Moi, quand j'ai commencé à sculpter dans l'atelier, dans les années 1961-1962, mon père était un petit peu en avance sur son temps. C'était à l'époque où mon père Médard faisait de la sculpture du nu. Alors l'atelier, à ce moment-là, était séparé en deux. Il y avait notre côté $^{81}$ et, de l'autre côté, mon père avait un genre de petite galerie d'art où il exposait ses œuvres. Mais les gens, souvent, ne rentraient pas là-dedans. Les gens de l'âge d'or, à l'époque, quand ils voyaient un nu, ils retournaient de bord : "On rentre pas là »; ils étaient scandalisés par ça. Alors mon père, lui, préférait s'en aller et c'est nous qui recevions les commentaires pas très gracieux. [Rires] [...] Mon père vendait ça à une certaine classe de gens, des médecins, des avocats, des notaires. Même il y a des femmes qui en ont acheté 82 . \ À l'époque où Médard commence à sculpter les nus, la pudibonderie dans la population en général ne permet pas de développer ouvertement cette forme d'art qu'il considère comme «l'art véritable », parce que l'homme et la femme représentent « ce que Dieu a créé de plus beau », mais combien difficile à représenter. Médard conclut : « Je laisserai que des morceaux de bois, mais je les aurai travaillés avec amour et satisfaction ${ }^{83}$. »

\section{Épilogue. Médard Bourgault}

Médard Bourgault est à l'origine d'un mouvement artistique authentique et identitaire, et le Québec s'est reconnu en lui. Son influence fut prégnante

77. Mмv 2008-0119, Entrevue d'André-Médard Bourgault par Guy Duguay, 7 octobre 2008.

78. Acs, F050/1/23, Correspondance, George O. Soucis, René-T. Leclerc Incorporée, Valeurs de Placement, Montréal, à Médard Bourgault, le 27 septembre 1955.

79. Acs, F050/1/25, Correspondance, Béatrice Ethier de Montréal à Médard Bourgault le 24 septembre 1957.

80. Acs, F050/1/27, Correspondance, et Acs, F050, E3 (F050/4/3), Cahier de vente de 1959, «Livré le 27 août 1959. »

81. La section de l'atelier où André-Médard sculpte avec Jacques, Fernand et Claude, JeanRaymond ayant son propre atelier.

82. Mmv 2007-0026, Entrevue d'André-Médard Bourgault par Martine Bélanger, 29 septembre 2007.

83. Médard Bourgault, Journal, op. cit., p. 104. 
chez les sculpteurs dont il a été le maître, sur les autres qui l'ont côtoyé et ceux qui ont suivi. Les journaux et les revues francophones et anglophones du Canada ont vanté le réalisme et l'authenticité de son œuvre. Il fut adulé par ses émules, recherché par le public et respecté par la critique. Médard Bourgault a eu seize enfants. Ses filles, qui ont sculpté avec lui, nous ont laissé des œuvres qui témoignent des travaux en atelier. Ses fils Jean-Raymond (1924-2010) ${ }^{84}$, Fernand (1932-1993), Claude (1933-1966), Jacques (1940$2017)^{85}$ et André-Médard (1941) sont tous devenus de grands sculpteurs ${ }^{86}$. Le dernier, André-Médard, par respect pour l'héritage reçu, a conservé intact le domaine érigé par son père qui est aujourd'hui un site patrimonial cité par la municipalité de Saint-Jean-Port-Joli ${ }^{87}$. Il a également publié avec François Gauthier La Maison de mon père, un livre généreusement illustré qui présente les bâtiments sur le domaine ainsi que les collections qu'ils contiennent ${ }^{88}$.

\section{André Bourgault (1898-1958)}

Le premier métier d'André Bourgault est celui de marin. Il navigue pendant une quinzaine d'années sur les barges des Grands Lacs. Comme il aime le dessin et la peinture, il profite d'un hiver, alors que le navire est accosté au port de Montréal, pour suivre des cours au Gesù ${ }^{89}$. Médard le rencontre par hasard à Québec en 1931 et il lui demande de venir sculpter avec lui et Jean-Julien : « Au mois de décembre, j'allai livrer à Québec quelques pièces que j'avais vendues à $\mathrm{M}^{\text {lle }}$ Corinne Méthot, employée au parlement. Je fis la rencontre de mon frère André, le décidai à venir travailler avec nous. Il avait une bonne partance dans la sculpture et le dessin. Nous revînmes chez nous ${ }^{90}$. [...] Nous nous mîmes au travail avec courage. André, après un peu de pratique, il vint à bout de faire une figurine. Alors ça alla bien. Au bout de quelque temps alors que j'étais à remplir une commande pour la Handicrafts Guild [1932], je fis faire une commande par mes frères, pour chez Holt Renfrew de Québec ${ }^{91}$.

84. Angéline Saint-Pierre, André Bourgault, op. cit., p. 73-87.

85. Voir ethnologiequebec.org/2017/03/jacques-bourgault-1940-2017/, consulté le 23 juin 2020.

86. Angéline Saint-Pierre, Médard Bourgault, sculpteur, Québec, Éditions Garneau, 1973, p. 117-128.

87. Le Domaine Médard-Bourgault a été cité par la municipalité de Saint-Jean-Port-Joli le 5 juin 2017 (Règlement 753-17) à la suite d'une demande de la part de la Société québécoise d'ethnologie. Voir aussi Benoit Vaillancourt, «Entre le père et l'artiste. Les héritiers de Médard Bourgault à Saint-JeanPort-Joli », Rabaska, vol. 17, 2019, p. 101-119.

88. Avec préface de Michel Lessard, Beloeil, Qualigram, 2015, XIX-156 p. Voir le compte rendu de Jean-François Blanchette, dans Rabaska, vol. 15, 2017, p. 229-233.

89. Angéline Saint-Pierre, André Bourgault, op. cit., p. 39.

90. Médard Bourgault, Journal, op. cit., p. 38.

91. W. MacMillan, Holt, Renfrew \& Co. Limited, Québec, place la commande à Médard Bourgault le 2 février 1932 (Acs, F050/1/2). Le 28 avril, il envoie ses commentaires : « Nous avons remarqué aussi que le dernier lot de statuettes reçues ne sont pas aussi soignées que le premier lot envoyé et nous vous demanderions de prendre plus de soin dans le découpage et aussi en ce qui regarde la peinture. » Malgré tout, il place une autre commande (Acs, F050/1/2). 
C'étaient de petites pièces de paysans ${ }^{92}$. »

Les frères sculptent ensemble pendant quelques années, puis André quitte Saint-Jean-Port-Joli en 1935 pour aller travailler avec les frères Bastien, spécialistes d'artisanat autochtone au Village-Huron ${ }^{93}$ près de Loretteville. Les frères Bourgault connaissent bien cette entreprise. En 1934 et $1935^{94}$, ils avaient eu d'eux plusieurs commandes de petites sculptures totalisant plus de mille pièces ${ }^{95}$. Un catalogue de la compagnie montre les produits que les Bastien vendent. Des figurines en bois des Bourgault y figurent ${ }^{96}$. André semble avoir influencé un sculpteur de Loretteville, Rosaire Audet ${ }^{97}$ qui aura son propre atelier et produira des figurines dans le style d'André et d'autres souvenirs qu'il développera pour le marché du souvenir ${ }^{98}$. André marie Cécile Lainé, huronne-wendat, à l'église Notre-Dame-de-Lorette de Wendake le 5 février 1936. Il revient la même année à Saint-Jean-Port-Joli avec sa nouvelle épouse. Comme André est un homme indépendant, personnellement et socialement, ils s'installent à l'extrémité du village, au Port Joly.

\section{L'atelier d'André Bourgault}

André Bourgault consacre toute sa carrière à l'art paysan, un style que ses deux frères vont peu à peu délaisser. Il sculpte des scènes de la vie rurale traditionnelle, de chasse et de pêche, les travaux des cultivateurs et des fermières, la vie de marin et des personnages autochtones. Il développe avec son beau-frère, Auguste Lainé, le concept de lampes surmontant un foyer devant lequel se bercent un bon vieux et sa vieille, avec leur chien et leur chat à leurs pieds ${ }^{99}$. Ce type de lampe, par la suite accompagné de diverses scènes de la vie traditionnelle paysanne, sera repris avec goût et variété par l'atelier de Paul-Émile Caron et d'autres. Il deviendra un objet type de la production locale.

André Bourgault est un habile sculpteur au canif qu'il manipule avec une habileté rare. Les médias aiment bien mentionner que la dextérité du

92. Médard Bourgault, Journal, op. cit., p. 38.

93. Les en-têtes de lettres indiquent «Indian Lorette », aujourd'hui Wendake. Voir F050/1/4, Correspondance, 1934-1935.

94. Médard raconte dans son Journal, op. cit., p. 38, que c'est en 1932 et 1933. Il a par erreur décalé ces dates comme celle de sa rencontre avec Marius Barbeau. Voir la référence à la correspondance dans la note suivante.

95. Acs, F050/1/4, Correspondance, Médard Bourgault à Marius Barbeau le 14 avril 1935.

96. Catalogue propriété de Richard Dubé.

97. La connaissance récente de cet artiste découle d'une exposition organisée par Charles Breton-Demeule en 2013. Voir Isabelle Chabot, "Rosaire Audet : un sculpteur prolifique oublié », www.quebechebdo.com/culture/98191/rosaire-audet-un-sculpteur-prolifique-o-3398378/, consulté le 23 juin 2020.

98. Voir également ce qu'en dit Jean-Marie Gauvreau, « Le Problème de l'artisanat ... », op. cit., p. $68-70$.

99. C'est l'avis d'André-Médard Bourgault. Il y a d'ailleurs, dans la collection du Musée de Charlevoix, une pièce signée par André et Auguste qui semble être un modèle de ces lampes. 
maître sort de sa tête, de ses mains et de ce seul outil. Ils associent cette technique à toute la production de Saint-Jean-Port-Joli, ce qui pourrait être un excès d'interprétation, quoique certains sculpteurs préfèrent le couteau : « Ils en possèdent plusieurs avec des lames différentes, selon les besoins. La souplesse de leurs gestes étonne les visiteurs ${ }^{100}$. » Par contre, les autres ont une panoplie d'outils à leurs dispositions et s'en servent pour accomplir les diverses étapes de production d'une pièce ${ }^{101}$.

Dès son retour à Saint-Jean-Port-Joli, André accepte dans son atelier des mains habiles - jeunes femmes et jeunes gens - qu'il encourage à produire des pièces pour son marché sans cesse croissant. Ils y trouvent, sinon un métier, du moins un travail d'appoint fort apprécié. Parlant de l'atelier de sculpture de Médard et Jean-Julien Bourgault qui ferme en 1942, Gérard Ouellet écrit : « Le gouvernement Duplessis reprendra l'initiative à l'atelier d'André Bourgault à l'automne 1944. ${ }^{102}$ » De fait, il ne s'agit pas d'une école proprement dite, mais d'un atelier où on forme des apprentis. Un programme gouvernemental leur permet de recevoir une aide pour se loger et apprendre le métier. Le sculpteur Marcel Guay, né en 1933, raconte qu'il y a droit de 1949 à 1953 comme élève d'André Bourgault : « Il nous appelait des élèves subventionnés. Les trois premières années, on avait une petite bourse. Le gouvernement nous donnait l'équivalent de deux piastres par jour, je pense. Ça payait notre pension. L'Aide à la jeunesse, qu'ils appelaient ça. [...]. André nous faisait faire des petits sujets qu'il pouvait vendre facilement : des coupepapiers, des têtes avec un bouchon en dessous, après ça, des petites figurines, là. Ce n'était pas de la grosse sculpture. Après plusieurs mois avec André, quand il a vu que ça fonctionnait comme ça puis que les pièces se vendaient, ça fait que là, il m'en faisait faire des plus grosses, un moment donné... 12 pouces, 24 pouces $^{103}$. » Angéline Saint-Pierre indique que des vétérans de la Deuxième Guerre mondiale reçoivent aussi cette aide pour y être formés ${ }^{104}$.

L'atelier-école d'André Bourgault du Port Joly qui était alors en pleine effervescence avec une vingtaine d'apprentis est détruit par le feu en 1945. Il y perd toute sa production et ses archives, ce qui oblige à examiner sa production et à utiliser les témoignages de ceux qui l'ont côtoyé pour documenter son histoire et sa production ${ }^{105}$. André reconstruit son atelier tout près de ses frères dès 1946 et il poursuit l'initiation d'apprentis avec l'aide de sa sœur Yvonne.

100. Alain Duhamel, Gens de bois, Saint-Jean-Port-Joli, Éditions Port-Joly, 1975, illustrations de Benoi Deschênes, p. 75

101. Sur le sujet de la sculpture sur bois, on lira avec intérêt Benoi Deschênes, La Gouge magique, Saint-Jean-Port-Joli, Éditions Port-Joly, 1988, 243 p.

102. Gérard Ouellet, Ma paroisse, op. cit., p. 302.

103. Mmv, Entrevue de Marcel Guay par Jean-François Blanchette, 6 juin 2017.

104. Angéline Saint-Pierre, André Bourgault.., op. cit., p. 42.

105. Sa biographe, Angéline Saint-Pierre, les a bien documentés dans André Bourgault, op. cit. 
La revue L'Ovale de CiL met en vedette l'atelier d'André en 1950 : «Le studio d'André occupe 20 personnes, dont 10 jeunes filles. En plus de sculpter les miniatures, celles-ci peignent les figurines de paysans offertes aux touristes. On compte huit étudiants employés en vertu d'un plan du gouvernement de la province de Québec pour favoriser le développement des arts et métiers ${ }^{106}$. \

Parmi les apprentis à l'atelier d'André on trouve, entre autres, ses fils Roger - devenu un grand sculpteur -, Jean-Guy et René, de même que Ferdinand Boucher, Antonio, Gertrude, Lucien et Madeleine Bourgault, Gisèle Caron, Agnès, Lionel et Simon Dubé, Ghislaine et Marguerite Gagnon, Marguerite Robichaud-Leclerc, Madeleine Mercier Ouellet, L. et Régina Normand, Adalbert Thibault, Laurent Fortin, Léon Toussaint, ainsi que Gérard Fortin et Marcel Guay qui deviendront des maîtres-sculpteurs et formeront d'autres apprentis, et bien d'autres. C'est sans aucun doute sous l'influence de tout ce village qui sculpte que la coiffeuse Jeanne Bélanger-Robichaud a pris goût à la sculpture, a sculpté et peint tout le village de Saint-Jean-Port-Joli, incluant son église qu'elle a produite en ex-voto pour faveur obtenue ${ }^{107}$.

Chacun sculpte avec raffinement et fins détails, les uns réussissant mieux que les autres. Ils signent souvent leurs pièces de leurs noms et André applique parfois un tampon sous la base des figurines "Élève André Bourgault ${ }^{108}$ ». En plus d'être un élément de fierté pour le maître et l'apprenti, cela est évidemment une approche gagnante pour la vente, car on veut du Bourgault. Comme la production appartient au maitre-sculpteur durant la période pendant laquelle l'apprenti reçoit l'aide du gouvernement, comme à l'atelier-école en 1940-1941, ce tampon en ferait foi. Les touristes sont très nombreux à s'arrêter à l'atelier d'André pour les voir travailler, lui et ses élèves, et acheter une pièce. Comme les touristes préfèrent les pièces peintes, les apprentis d'André et lui-même continueront d'ajouter de la couleur aux pièces, car cela « leur donne de la vie ». Mais une tendance qui commence à s'installer, depuis l'atelier-école de 1940-1942, est de laisser les œuvres au bois naturel, surtout les grosses pièces, ce qui les distingue du plâtre peint, aux yeux de Jean-Marie Gauvreau qui regarde de près ce qui est produit dans les ateliers et qui de plus recommande l'aide financière aux jeunes.

Depuis les débuts, on peut voir des numéros sur les pièces d'André, sans doute des numéros de séries. On standardise la production en répétant les œuvres afin de répondre à la demande de ce que le marché préfère. C'est d'ailleurs ce que Marius Barbeau avait suggéré à Médard en 1933 : «Il n’y a

106. Marge et Phil Shackleton, « Au pays des sculpteurs », L'Ovale CIL, août 1950, p. 8-9.

107. Cette œuvre d'art populaire majeure de 262 pièces est désormais au Musée canadien de l'histoire à Gatineau. Jean-François Blanchette, Du coq à l'âme, op. cit., p. 219, et www.museedelhistoire. $\mathrm{ca} / \mathrm{b} \log /$ toute-une-epoque-en-sculpture-le-village-en-miniature-de-saint-jean-port-joli.

108. On trouvera de nombreux exemples dans la collection du Musée de Charlevoix. 
pas de mal à ce que vous répétiez vous-même vos meilleurs modèles si vous les placez dans des villes différentes. Même le commerce exige cela, que vous fassiez des choses en séries, c'est-à-dire répétez plusieurs fois le même sujet, petit, et à bon marché ${ }^{109}$.» Les grands magasins et distributeurs préfèrent une standardisation, mais les responsables de l'inventaire artisanal indiquent que cela nuit à la créativité ${ }^{110}$. Malgré cela, les journaux de l'époque mentionnent la qualité des travaux qui sortent de Saint-Jean-Port-Joli : «Depuis 1927, des milliers de statuettes sont sorties des mains des Bourgault. Elles sont dispersées aux quatre coins de l'Amérique. Aucune ne porte le caractère banal des travaux en série ${ }^{111}$. » La valeur de l'artisanat produit à Saint-Jean-Port-Joli est imposante. Jean-Marie Gauvreau rapporte que la seule compagnie Robert Simpson Co. Ltd a commandé pour 37260 \$ de pièces d'artisanat de la région entre 1940 et $1943^{112}$. En valeur de 2019, cela équivaut à $578376 \$$ pour ce seul marchand ${ }^{113}$. Gauvreau témoigne, en juin 1947, que « les Bourgault par leur opiniâtreté et leur travail, ont doté Saint-Jean-Port-Joli d'une industrie qui emploie 60 artisans initiés à la sculpture sur bois ${ }^{114} \gg$ dont le revenu est évalué à $150000 \$ 11$.

\section{Le Centre d'artisanat enr., 1953-1958}

En 1953, malade et incapable de s'occuper de ses affaires, André laisse le contrôle de son entreprise et les droits de vente de sa production à un entrepreneur local, Maurice Leclerc. Le maître-sculpteur André Bourgault est désormais l'employé de l'homme d'affaires ${ }^{116}$. C'est une période sombre, malgré les photos et les reportages publicitaires. Sa biographe Angéline Saint-Pierre ${ }^{117}$ choisit de ne pas en parler, sans doute pour ne pas conserver la mémoire de ces tristes jours. André décède, frappé par une voiture, le 31 janvier 1958. Sa grande collection de pièces non vendues demeure la

109. Acs, F050/1/3, Correspondance, Marius Barbeau à Médard Bourgault, 13 juin 1933.

110. Au sujet de la demande de standardisation, voir la lettre de L. Arsenault du ministère des Affaires municipales, de l'industrie et du commerce à Jean-Julien et Médard Bourgault le 29 mai 1941 (Acs F050/1/9). Par contre, les enquêteurs de l'inventaire artisanal du comté de Kamouraska s'en plaignent (BANQ, MSS2/6/12).

111. "Les Bourgault, chantres de la paysannerie », La Patrie, 22 septembre 1940.

112. Archives du CÉGEP du Vieux-Montréal, Fonds de l'École du meuble, EM-10.7.12, lettre de Jean-Marie Gauvreau à Oscar Drouin, 21 octobre 1943.

113. Selon la feuille de calcul de l'inflation de la Banque du Canada entre 1942 et 2019, consultée le 23 juin 2020 : www.banqueducanada.ca/taux/renseignements-complementaires/feuille-de-calcul-delinflation.

114. BANQ, Fonds Jean-Marie Gauvreau, MSS2/11/4, « Exposition provinciale de l'artisanat », Sherbrooke, juin 1947.

115. BANQ, MSS2/11/5.

116. Association des artisans de Saint-Jean-Port-Joli Inc., Collection d'œuvres, Montréal, La Compagnie Rothmans de Pall Mall Canada Limitée, 1973. Catalogue d'exposition, s. p. Voir la rubrique «André Bourgault ».

117. Angéline Saint-Pierre, André Bourgault, op. cit. 
propriété de Maurice Leclerc qui vendra le Centre d'artisanat enr. à Berthier Beauregard en 1978 et créera un musée de la sculpture sur bois, le Musée des Anciens Canadiens ${ }^{118}$.

\section{La notoriété d'André Bourgault}

Le marché d'œuvres pour le tourisme n'empêche pas la production de pièces majeures ; bien au contraire, il le favorise, car cela fait connaître le maîtresculpteur de sorte que son studio attire collectionneurs et clients réguliers ${ }^{119}$. Le talent d'André est reconnu très tôt et on le réclame pour des expositions et des démonstrations, comme c'est le cas en 1941 alors que la maison Morgan l'invite à sculpter devant le public pendant une semaine à son magasin de Montréal ${ }^{120}$. Le sculpteur reçoit des commandes du Canada, des États-Unis et même d'Europe. On désire des pièces imposantes pour offrir en cadeaux aux personnages importants qui visitent le Québec et le Canada. Ainsi, à titre d'exemple, André Bourgault est un invité de marque à l'exposition artisanale tenue au Château Laurier, à Ottawa, du 6 au 11 mars 1950. Cet événement est organisé par l'Office provincial de l'artisanat et de la petite industrie, en collaboration avec la Fédération des chambres de commerce des jeunes de la Province de Québec. La Presse, The Gazette du 8 mars et de nombreux autres journaux rapportent l'événement et publient des photos d'André et des invités avec ses œuvres. À cette occasion, une sculpture d'un labour avec bœufs attelés signée par l'artiste est offerte à $\mathrm{M}^{\mathrm{me}}$ Louis Saint-Laurent, épouse du premier ministre du Canada ${ }^{121}$.

\section{Épilogue. André Bourgault}

L'influence d'André Bourgault est grande, car il a formé de nombreux apprentis qui ont pu vivre de ce métier ou en tirer un revenu d'appoint. Il a fait œuvre de mémoire en racontant dans le bois la vivacité de la vie traditionnelle des Québécois sur terre et en mer. Pierre Bourgault fait l'éloge de son oncle André : «André était le seul qui faisait impression et non réalité. Tu sais, pour André, qu'il y ait trois doigts ou cinq doigts, ça lui importait peu. Il faisait comme les impressionnistes, ce que tu voyais de loin, tandis que Jean-Julien et Médard faisaient le réel. C'était une grosse différence. André était d'abord peintre. Il avait une âme d'artiste très profonde. Il faisait ce que tu vois quand tu es de loin, tandis que Jean-Julien et Médard essayaient de représenter la réalité. C'est ça, la grosse différence.

118. Ce musée, qui raconte l'histoire de la sculpture à Saint-Jean-Port-Joli, expose près de 400 œuvres d'une centaine de sculpteurs.

119. Marge et Phil Shackleton, op. cit., p. 8-9.

120. La Presse, 10 mai 1941, p. 41, cité par Angéline Saint-Pierre, André Bourgault, op. cit., p. 36 et 48 .

121. BANQ, MSS2/11/6. 
Les sculptures de mon oncle André sont merveilleuses. Tu regardes ce violoneux, là, tu entends la musique ${ }^{122}$. » André Bourgault a eu onze enfants avec Cécile Lainé. Parmi les garçons qui ont pratiqué la sculpture, RogerAndré deviendra un grand sculpteur de renom, versatile tant dans la sculpture traditionnelle que moderne ${ }^{123}$.

\section{Jean-Julien Bourgault}

Magloire envoie étudier son fils Jean-Julien à Montréal alors qu'il est âgé de 12 ans, mais ce dernier s'ennuie et il revient dans son patelin : " Mon père m'a alors laissé le choix de prendre un autre chemin que les études tout en m'informant de choisir le bon chemin ${ }^{124}$. » Après avoir assisté son oncle Antonio Bourgault, gardien du phare au Pilier de pierre en 1925-1926 ${ }^{125}$, il s'engage en 1926-1927 et 1928-1929 comme marin à bord du Sable I ${ }^{126}$. En 1928-1929, il suit des cours d'ébénisterie et d'anglais à Québec, et accepte ensuite des contrats de menuiserie ; mais il est obligé de revenir dans son patelin en raison de la crise économique et il travaille à l'atelier de menuiserie avec son père et son frère Médard ${ }^{127}$. Ce dernier le convainc de l'aider à remplir ses commandes de sculptures en $1931^{128}$.

\section{Jean-Julien, sculpteur}

Jean-Julien sculpte à temps complet en 1932 et les trois frères ont tellement de travail que leur père les aide financièrement pour la construction d'un atelier en $1933^{129}$. " C'est un vrai studio des frères Bourgault, à qui ferait la pièce la plus originale et ça devient que l'on ne trouve plus de vrais moments de $\operatorname{repos}^{130} »$, écrit Jean-Julien.

Comme Jean-Julien est habile à manier le ciseau et qu'il aime la composition, il réussit très vite à produire des œuvres qui plaisent à la clientèle. Médard étant un homme réservé, c'est Jean-Julien qui anime les rencontres avec le public. Il aime les échanges enjoués avec ceux qui visitent leur atelier. Il est généreux et il accueille facilement ceux qui se cherchent

122. Mmv, Entrevue de Pierre Bourgault par Jean-François Blanchette, 5 juin 2017.

123. Angéline Saint-Pierre, André Bourgault, op. cit., p. 73-87.

124. Acs, Fonds Jean-Julien Bourgault, F215/1/4, Notes biographiques dactylographiées datées du 14 octobre 1961.

125. Acs, F215/1/4, Notes manuscrites.

126. Loc. cit.

127. Acs, F215/1/4, Notes manuscrites autobiographiques. Acs, F001, D46, Municipalité de Saint-Jean-Port-Joli, « Jean-Julien Bourgault, artiste sculpteur, Saint-Jean-Port-Joli » (brève biographie rédigée par Jacques Castonguay « pour Jean-Julien Bourgault » le 17 mai 1978).

128. Acs, F215/1/4, Notes manuscrites autobiographiques.

129. Cet atelier a été détruit en décembre 2016 : «Un grand symbole s'en est allé... » medardbourgault.org/2016/12/19/un-symbole-sen-est-alle/amp, consulté le 23 juin 2020. Voir aussi Acs, Fonds Jean-Julien Bourgault, F215/1/4, Notes manuscrites autobiographiques.

130. Acs, F215/1/4, Notes biographiques. 
un toit. Homme pragmatique, il réussit bien en affaires. Comme pour ses frères, c'est l'art paysan qui a été la base du marché rapide qu'il a réussi à développer au début des années 1930 et « ses statuettes pourraient constituer un musée fort expressif des travaux et de l'âme de nos gens. ${ }^{131} \gg$ De plus, comme il est bon en ébénisterie, c'est lui qui réalise les pièces de mobilier d'église quand Médard reçoit des commandes qui requièrent cette expertise.

\section{L'atelier de mobilier d'église de Jean-Julien Bourgault, 1949-1957}

Jean-Julien profite, avec son frère Médard, du renouveau de ferveur religieuse qui dure depuis le milieu du XIX ${ }^{\mathrm{e}}$ siècle ${ }^{132}$ et qui se poursuit jusqu'au milieu $\mathrm{du} \mathrm{XX}^{\mathrm{e}}$ siècle. Les communautés religieuses, les paroisses et les cathédrales du Canada commandent des sculptures religieuses de Médard dès leur début et Jean-Julien l'assiste ${ }^{133}$. En 1937 en effet, les deux frères sculptent la chaire de l'église de leur paroisse qui leur commandera d'autres pièces plus tard ${ }^{134}$. Comme la demande pour le mobilier d'église est grande, Jean-Julien quitte l'atelier qu'il partage avec Médard et fonde un atelier qui se spécialise dans ce domaine : « Je commence un atelier de sculpture et ameublement d'église pour initier mon fils Gilles et le préparer à un avenir. Je dirige ce genre de travail pendant 8 années : sculpture, dessin, architecture, ébénisterie, etc. ${ }^{135}$ । Jean-Raymond, le fils de Médard qui revient de quatre années (1945-1949) de formation à l'École des beaux-arts de Québec où il s'est spécialisé en art décoratif, se joint à eux et il y sera jusqu'en $1956^{136}$. Jean-Julien engage des sculpteurs comme Jean-Marc Deschênes, Denis Guimont, Max Kutzera, Wilfrid Richard et Albert Nadeau ${ }^{137}$. Ce dernier a passé huit années à l'atelier d'art décoratif de Leonardo Ottino de Montréal où il a développé ses talents en ce domaine. Il rejoint l'atelier de Jean-Julien en 1954 afin de l'aider à améliorer les techniques de production ${ }^{138}$. De nombreux menuisiers complètent les équipes de travail, sans compter les apprentis comme Marcel

131. La Patrie, 22 septembre 1940

132. Diane Saint-Pierre, « Un nouvel encadrement institutionnel. 1850-1930», dans Alain Laberge (dir.), Histoire de la Côte-du-Sud, Québec, Institut québécois de recherche sur la culture, « Les régions du Québec » 4, 1993, p. 288.

133. Gérard Ouellet indique en 1944 que Jean-Julien assiste Médard dans la production d'art religieux qui est devenu la spécialité de ce dernier (G. Ouellet, Ma paroisse, op. cit., p. 302).

134. Nicole Bourgault et Jean Simard, op. cit.

135. Acs, Fonds Jean-Julien Bourgault, F215/1/4, Notes biographiques dactylographiées datées du 14 octobre 1961.

136. Nicole Bourgault, Hélène Bourque, Claude Corriveau et Ginette Laroche, Le Patrimoine religieux de l'église Saint-Charles-Garnier, Québec, Fabrique Saint-Charles-Garnier, 2012, p. 36.

137. Nicole Bourgault, « EEuvres de sculpture et d'ébénisterie de l'église Saint-Charles-Garnier comprenant une analyse particulière de l'atelier Jean-Julien Bourgault», Rapport de recherche, Québec, Ville de Québec et Fabrique Saint-Charles-Garnier, mai 2010, p. 70.

138. Mmv 2008-0122-MF, Entrevue de Robert Roy par Guy Duguay, 8 octobre 2008. 
Guay qui deviendra par la suite maître-sculpteur à son propre atelier ${ }^{139}$.

Il y a création d'un patrimoine religieux considérable. Le style de l'ameublement et du décor d'église qui est adopté s'inspire de celui de l'école Boulle de Paris où Jean-Marie Gauvreau a étudié ; Nicole Bourgault le montre en publiant un dessin de l'école annotée par Jean-Julien ainsi que la liste de livres de cette école que son père a dans sa bibliothèque ${ }^{140}$. On crée l'ameublement et le décor pour 70 cathédrales, églises, chapelles et monastères du Québec, du Canada et des États-Unis. Il y a peu de documentation écrite sur le détail des réalisations de mobilier d'église de Jean-Julien Bourgault. La richesse artistique et architecturale de l'église Saint-Charles-Garnier qui, de fait, porte la signature de Jean-Julien Bourgault, a par ailleurs et fort heureusement été analysée par l'équipe mise en place par Louise Lafrance pour la fabrique de la paroisse et présentée dans le magnifique album Le Patrimoine religieux de l'église Saint-CharlesGarnier $^{141}$ qui « contextualise l'histoire et l'architecture de l'église bien sûr, mais documente aussi finement les œuvres d'art - sculptures, verrières et mosaïques, entre autres - serties dans le somptueux décor néogothique de pierres, de boiseries et de granit. La modernité et la tradition s'y côtoient dans des œuvres d'art de ces dernières décennies où l'on magnifiait encore le divin au Québec ${ }^{142}$. » En effet, les sculptures et meubles suivants ont été sculptés et fabriqués par le maître-sculpteur entre 1949 et 1953 : les boiseries et les stalles du chœur, le tombeau du maître-autel, la chaire de la nef, le transept, le retable et l'ambon du chœur, le cierge pascal et les cierges porteflambeaux, ainsi qu'un magnifique tabernacle en acajou, considéré comme une œuvre maîtresse ${ }^{143}$. Le corpus du maître-autel est l'œuvre de Médard Bourgault. On peut conclure, de concert avec les promoteurs du patrimoine paroissial, que « le patrimoine artistique de l'église correspond ainsi à une époque charnière de l'histoire de l'art qui voit se renouveler l'art religieux, avant le modernisme des années $1960^{144}$. » Il constitue de plus l'ensemble le mieux conservé de la contribution de Jean-Julien Bourgault au patrimoine religieux ${ }^{145}$.

Malgré les nombreuses réalisations et les commandes existantes, Jean-Julien doit ralentir la production de mobilier d'église en raison de la

139. Mmv, Entrevue de Marcel Guay par Jean-François Blanchette, 6 juin 2017.

140. Nicole Bourgault, « Euvres de sculpture et d'ébénisterie... », op. cit., p. 41-42, 64 et 67.

141. Nicole Bourgault et al., Le Patrimoine religieux de l'église Saint-Charles-Garnier, op. cit.

142. Jean Simard, ethnologiequebec.org/2012/11/le-patrimoine-religieux-de-leglise-saint-charlesgarnier, consulté le 21 juin 2020.

143. Le tabernacle, le cierge pascal, les cierges porte-flambeaux, ainsi que d'autres pièces ont été exposés dans "Trésors de Saint-Charles-Garnier» à la Villa Bagatelle. Voir www.quebechebdo.com/ culture/122038/tresors-de-saint-charles-garnier-a-la-3634322, consulté le 20 juin 2020.

144. paroisse-saint-charles-garnier.org/paroisse-stc/historique-etc, consulté le 21 juin 2020.

145. Nicole Bourgault, «CEuvres de sculptures et d'ébénisterie... », op. cit., p. 62. 
compétition féroce des marchands de plâtre du Québec et d'Italie et des ébénistes locaux et internationaux qui sont fort présents sur les marchés ${ }^{146}$.

\section{L'" École de sculpture Jean-J. Bourgault ", 1957-1967}

Le ralentissement de la production à l'atelier de mobilier religieux amène Jean-Julien à se concentrer sur la sculpture proprement dite. C'est une décision qu'il a mûrement réfléchie au cours des dernières années : « Mais je travaille toujours avec le désir de retourner à mon studio personnel ainsi que Gilles avec la même ambition ; je décide donc un bon matin en 1957 de discontinuer ce métier afin d'avoir la liberté de créer d'autres œuvres. Je commence donc [...] une école de sculpture pour une vingtaine d'élèves $[\ldots]^{147}$. " Cette fois-ci, par contre, Jean-Julien est assisté par son épouse Marie-Antoinette Caron, aux fonctions administratives, et son fils Gilles, désormais sculpteur professionnel. Cette unité familiale constitue le noyau de l'atelier-école et en permet le succès.

Robert Rice de la Presse canadienne écrit dans L'Action catholique du 26 février 1958 : « Le gouvernement provincial lui a aidé à établir une école artisanale où il transmet son art à des élèves. À l'heure actuelle, il prend sous sa tutelle, dans son atelier, de jeunes artisans au talent certain sans qu'il leur en coûte rien. Dans cet atelier, il dispose d'une panoplie de plus de 250 outils de travail et utilise surtout les bois du Québec dans ses travaux de sculpture. Il importe, toutefois, de l'acajou de l'Amérique du Sud, employant les bois les plus vieux, partant les plus secs et les plus propices à la sculpture. [...] Le prix des ouvrages de Jean-Julien varie entre 50 cents à $2000 \$$ par sculpture. Ses œuvres sont disséminées de par le monde ${ }^{148}$. » Pour sa part, Jacques Trépanier rapporte, dans La Patrie du dimanche du $1^{\mathrm{er}}$ novembre $1959^{149}$, que Jean-Julien Bourgault a ouvert un atelier de sculpture appelé « Les Ateliers J.-J. Bourgault », « à la suggestion de M. Jean-Marie Gauvreau, directeur de l'Artisanat et de la petite industrie. Les ateliers sont ouverts depuis un mois et opèrent sous l'égide du Service des cours de culture populaire du ministère de la Jeunesse. " Il y mentionne les onze élèves alors inscrits : «Claudette, fille de Jean-Julien Bourgault, Roger et René, deux fils d'André Bourgault (frère de Médard et Jean-Julien) décédé il y a deux ans ; Claude,

146. Les références sont nombreuses. En voici quelques-unes : Acs, F050/1/10, Médard Bourgault à Albert Tessier le 19 mai 1942 ; Acs, F050/1/26, J. Michaud, Casavant Frères Limitée, à Médard Bourgault, 27 décembre 1958 ; Paul Trépanier, «Le sculpteur Henry Angers : la fin d'une tradition », Cap-aux-Diamants, vol. 3, n 1, 1987, p. 84.

147. Acs, F215/1/4, Notes biographiques dactylographiées datées du 14 octobre 1961. JeanRaymond décide la même année de quitter l'atelier de Jean-Julien et de créer son propre atelier.

148. L'Action catholique, février 1958, coupure, Acs, F100/389/61. Cet article est également publié dans La Tribune du 27 février 1958, p. 11, et dans Le Soleil du 26 février 1958, p. 3.

149. Jacques Trépanier, La Patrie du dimanche, $1^{\text {er }}$ novembre 1959, p. 116. 
Jacques et André, fils de Médard. Des élèves viennent de l'extérieur, comme Victor Dallaire de Grande-Baie, Saguenay ; Fernand Caron et Lucien Dubé de Saint-Albert de L'Islet. De Saint-Jean-Port-Joli, encore Lucien Picard et Robert Perreault, ce dernier [étant] le fils de Gérard Perreault, artiste peintre de Montréal dont la famille demeure pendant neuf mois à Saint-JeanPort-Joli ${ }^{150}$. » Dans le même article, il décrit les conditions de l'entente avec le ministère et les responsabilités vis-à-vis des apprentis. Les termes sont similaires à ceux que le ministère avait négociés avec Médard en 1953 et qui ont été mentionnés plus haut.

L'approche pédagogique demeure la même que celle établie dès les premiers ateliers, une formation pratique à la sculpture sur bois en taille directe. Les élèves - jeunes gens et aussi jeunes filles - viennent de partout au Québec. Les pièces produites appartiennent à l'école qui les vend. Gaétan Hovington raconte son apprentissage chez Jean-Julien alors qu'il y reçoit l’Aide à la jeunesse de 1962 à 1964 :

Étant jeune, j'avais toujours un couteau de poche puis je faisais plein de petites choses. Puis dans les années 1960, le curé de la place ici avait vu que j'aimais plus travailler avec le couteau de poche que d'aller à l'école, ça fait qu'il m'avait suggéré d'apprendre la sculpture. Ce curé-là allait à la pêche au lac Jacques où il rencontrait Médard et Jean-Julien Bourgault. Les curés, dans les années 1960, c'était puissant, c'était eux autres qui dirigeaient pas mal le monde. Ça fait que c'est de là que ça a parti et le curé m'avait inscrit à l'école de Jean-Julien Bourgault en 1962. J'avais 16 ans. À l'école, c'était quand même bien organisé pareil pour les années. Le gouvernement subventionnait Monsieur Bourgault pour donner des cours et puis les pièces qu'on faisait restaient à l'école. On avait aussi un petit salaire. Le gouvernement nous octroyait 18 dollars par semaine. On restait en pension dans des familles, ce qui nous coûtait 14 dollars par semaine, logé et nourri.

L'apprentissage ne se faisait pas dans les livres comme dans les écoles d'aujourd'hui où on apprend l'histoire de l'art, puis tout ça, là. Ce n'était pas la grande théorie. C'était vraiment du travail d'atelier. On arrivait à l'atelier le matin à 8 heures puis on travaillait jusqu'à 5 heures. On était plusieurs jeunes artisans autour d'une grande table puis monsieur Bourgault passait à différentes heures pour faire le tour de tous les élèves, tous les artisans, puis là il disait : "Bien ça, fais pas ça de telle façon. "Ça fait que là, il prenait un outil : "Bien, regarde, ça, je vais te corriger ça. "Il arrivait puis il le corrigeait. Ça fait que c'était de cette façon-là qu'il nous montrait. Puis je pense que c'était la façon la plus simple, puis c'était assez rapide aussi, parce qu'on ne perdait pas de temps, c'était de l'ouvrage d'atelier. Ça partait du principe "C'est en forgeant que tu deviens forgeron ».

150. Loc. cit. 
À l'école, bien, on avait des thèmes quand même pour nous faciliter le travail. On commençait par le maniement des outils ; on sculptait des objets plus faciles au début comme creuser des plats en forme de feuille d'érable, par exemple. Ça durait une petite période de temps. Après ça, on touchait le bas-relief, les profondeurs de champ, bien calculer nos angles. Par après, la ronde bosse. Ça, c'était plus difficile. On apprenait toutes ces choses-là avec l'expérience de ces gens-là ${ }^{151}$.

Dans leur présentation de l'histoire de la sculpture à Saint-Jean-Port-Joli, Alain Duhamel et Benoi Deschênes ne manquent pas de mentionner que les femmes, qui sculptent aux ateliers des hommes ou chez elles comme travail d'appoint, au début des années 1930, sont nombreuses à avoir leurs propres ateliers à partir des années $1960^{152}$. Ils pointent en effet vers le magnifique volume Mains habiles... Mains agiles ${ }^{153}$, dans lequel plusieurs femmes sont présentées au travail dans leurs ateliers. Il faut ajouter qu'elles s'occupent aussi de fonctions administratives quand elles sont responsables des magasins, de la gestion des livres de vente et de compte, ainsi que de la correspondance.

L'École de sculpture Jean-J. Bourgault ferme au printemps 1967. Cet atelier-école aura eu un impact majeur sur la sculpture sur bois en taille directe, car on y a formé près de 200 sculpteurs dont plusieurs ont vécu et vivent toujours de leur art. Parmi les élèves de Jean-Julien au cours de ces années, on trouve de nombreux sculpteurs qui ont fait leur marque dont Pier Cloutier, Maurice Harvey, Denys Heppell, Gaétan Hovington, André Pelletier, Herman Raby, et bien d'autres. Certains d'entre eux comme Gil-Jacques Cloutier, Victor Dallaire, Benoi Deschênes ${ }^{154}$, Nicole Deschênes Duval et Marcel Guay sont devenus maîtres d'ateliers et ont formé d'autres apprentis. Au cours des années, la réputation des sculpteurs formés à SaintJean-Port-Joli a eu un attrait irrésistible de la part de nombreux artistes en art populaire du Québec qui ont suivi leur exemple, adopté leur style et pratiqué cette forme d'art ${ }^{155}$.

\section{L'art de Jean-Julien Bourgault}

Talentueux en dessin ${ }^{156}$, Jean-Julien l'est également pour exprimer dans le bois les caractères de ses personnages. Il est un grand observateur de ce

151. Mмv, Entrevue de Gaétan Hovington par Jean-François Blanchette, 13 juin 2017.

152. Alain Duhamel, Gens de bois, op. cit., p. 66.

153. Maurice Marquis, Mais habiles... Mains agiles, Montmagny, Éditions Marquis Ltée, [1966], 133 p., nombreuses illustrations.

154. Benoi Deschênes, Benoi Deschênes, sculpteur et peintre, Saint-Jean-Port-Joli, Éditions Port-Joly, 2011, 239 p.

155. Comme j'ai pu le constater au cours de mes travaux de recherche sur le terrain.

156. Sa production en dessin est imposante. On en trouve une belle collection aux Acs, F215 E. 
qui se passe autour de lui. Il aime représenter dans le bois, souvent avec humour, les activités sociales, religieuses et politiques qui ont lieu dans la communauté, les assemblées publiques à la sortie de l'église ou au marché. Ses bas-reliefs, ses rondes bosses et ses pièces montées présentent ainsi ses concitoyens avec des allures cocasses. La vivacité de ses rondes bosses exprime les états d'âme des humains, souvent de ses contemporains, amis, voisins et personnages historiques. Son humour et sa critique trouvent place dans les scènes de ses bas-reliefs grâce à son style caricatural. Son amour de la culture orale locale lui permet de représenter son imaginaire avec exubérance. Contes, légendes et récits fantastiques se retrouvent exprimés dans des rondes bosses et des bas-reliefs savoureux, comme $L a$ Chasse-galerie et La Coureuse des grèves, imprégnés d'une interprétation toute personnelle. Enfin, son amour de la nature lui donne des occasions de s'éloigner de ses tâches et l'inspiration qu'il y puise renouvelle son impulsion créatrice. Le sculpteur Jocelyn Bouchard raconte le ressourcement de Jean-Julien Bourgault dans la nature :

Jean-Julien m'encourageait à créer puis à observer, écouter la nature aussi. Une fois, Jean-Julien et moi, on part, on s'en va dans les montagnes. On se met à marcher, puis tout le temps qu'on marchait, je ne sais pas pourquoi, on ramassait les fleurs. Il dit "Petit, je suis fatigué, moi ». "On a le temps, grand-père, assoyez-vous ». On s'assit sur un billot. D'un coup, on s'allume une cigarette, on fume tranquillement, on ne se parlait pas. On regardait, on observait. - Ça fait que Jean-Julien, lui, il avait une manie. Il avait plein de petits crayons partout. Quand il en avait un neuf, il le cassait en deux, il en faisait deux avec. Des petits bouts de papier, aussi, tu sais. Jean-Julien avait même dessiné sur le poignet de sa chemise blanche un dimanche à la messe [Rires]. C'était continuel. - Ça fait qu'on était là, on observait. Il sort son petit papier et son petit crayon, il commence à griffonner. Puis là, ce qu'on voit apparaître : une belle madone à la Jean-Julien, toute torsadée. Je regarde la plante et je m'aperçois que les lignes correspondaient avec les lignes de la madone. Ça, c'est une grosse plante qu'on appelle le chou puant. C'est une grosse fleur violacée qui pue. La créativité, elle vient de là. On n'invente rien, hein ?157

\section{Épilogue. Jean-Julien Bourgault}

Jean-Julien Bourgault, plus que tout autre sculpteur de son époque, a compris le besoin des Québécois d'exprimer profondément ce qu'ils sont. Sa très grande capacité de communication et d'échange avec ses interlocuteurs lui a permis de créer des œuvres qui témoignent de son époque, en même temps qu'elles sont intemporelles, car elles expriment des états d'âme profondément humains. Maître-sculpteur, il le fut. Non seulement en raison de la qualité et

157. Mmv 2014-0079, Entrevue de Jocelyn Bouchard par Judith Douville, 3 septembre 2014. 
de la créativité de son œuvre, mais également parce qu'il a formé un nombre imposant d'apprentis tout au long de sa carrière. Son influence fut immense. Jean-Julien Bourgault est décédé en 1996, laissant derrière lui sa famille et ses élèves, mais aussi, dit-on, un carnet de commandes plein. L'œuvre de Jean-Julien Bourgault aura été remarquable et abondante ${ }^{158}$. Il aura raconté la vie autour de lui avec beaucoup d'acuité et d'humour. Il aura meublé nos lieux de culte afin que l'action de l'homme soit visible et tangible, là où ce dernier se rassemble pour honorer son Dieu. Il aura été un artiste de son temps, sculptant le laboureur et l'homme religieux de son époque pour s'adonner plus tard au culte du nu féminin dans toute sa sensualité.

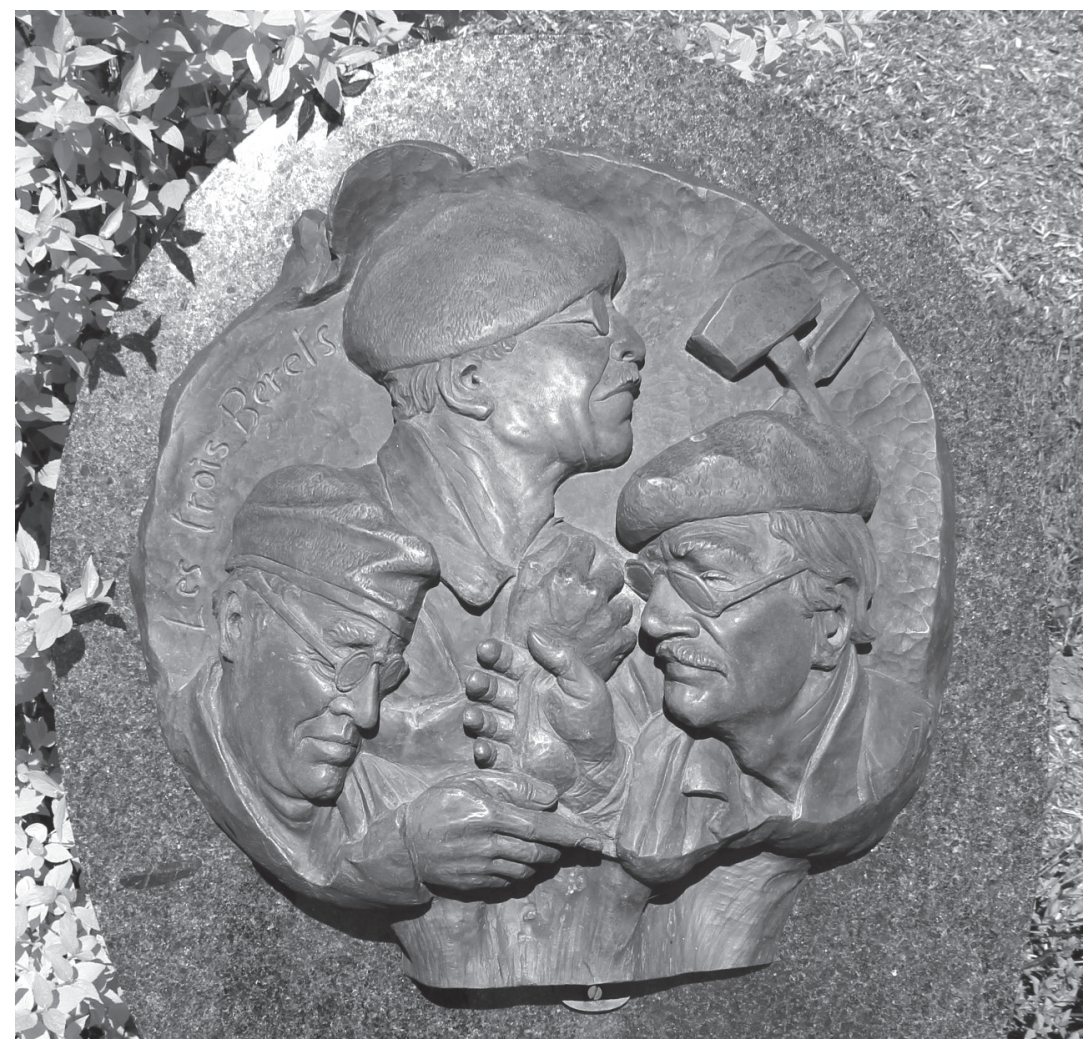

\section{Les Trois Bérets}

Bronze réalisé par Roger-André Bourgault à partir d'une sculpture de son oncle Jean-Julien Bourgault. Offert par les donateurs du Fonds d'acquisition d'œuvres d'art de Saint-Jean-Port-Joli, il fut inauguré le 24 juin 1993 et placé devant l'hôtel de ville.

Photo : Jean-François Blanchette, 26 mai 2016

158. Comme la période de cet article se termine en 1967, avec le décès de Médard, il y a un pan important de l'œuvre de Jean-Julien qui n'est pas couvert ici. 


\section{V. Épilogue général}

Les trois frères Bourgault ont formé des apprentis toute leur carrière durant et le style de ces derniers est imprégné de celui de leurs maîtres. Ils n'ont pas ménagé leur encouragement ni leur temps et ont agi en véritables porteurs de traditions. On leur doit plusieurs générations de nouveaux sculpteurs qui se sont établis à Saint-Jean-Port-Joli ou sont retournés dans leur patelin après leur formation ${ }^{159}$.

\section{Saint-Jean-Port-Joli, capitale de la sculpture sur bois}

Le mouvement de sculpture sur bois en taille directe de Saint-Jean-Port-Joli a près d'une centaine d'années. Il a pris ses sources dans l'art populaire traditionnel des Québécois et s'est développé en une forme esthétique nouvelle, originale, authentique et identitaire. Il n'est pas possible de le classer dans les catégories habituelles de l'art, de l'artisanat ou de l'art populaire. Les trois maîtres sculpteurs à l'origine de ce mouvement étaient autodidactes. Ils ont été influencés au cours de leur carrière, et dans le raffinement de leur art, par les beaux-arts et le marché touristique, mais ils sont demeurés authentiques et loyaux à leurs racines. Finalement, il faut réaliser qu'ils se sont développés en un " réseau parallèle ${ }^{160}$, aux dires même de Pierre Bourgault, fils de Jean-Julien. Les Trois Bérets étaient trop près de leurs travaux pour se rendre compte qu'ils ont créé un style original qui a fait école et qui est demeuré unique en Amérique du Nord. Dès 1932, on reconnaissait Saint-Jean-Port-Joli comme « la patrie de nos sculpteurs sur bois ${ }^{161}$ » en raison de leurs travaux et le titre de « capitale de la sculpture sur bois » est attribué au village lorsque Médard reçoit le prix de l'artisanat du Québec en 1953. Les frères Bourgault et leurs émules ont fait l'objet de nombreux articles et reportages, dont plusieurs fort prestigieux. Mentionnons, à la fin de la période couverte par cet article, celui que la revue Pace de Californie publie en 1967, intitulé « The modern artisans of St. Jean Port Joli ${ }^{162}$ », dans lequel on fait écho à l'histoire de ce mouvement unique ainsi qu'à toute la vigueur dont il est empreint; et enfin celui d'Yves Dubé publié dans la revue Forces d'Hydro-Québec, « Médard Bourgault :

159. Dix sculpteurs héritiers des Bourgault ont été honorés par la Société québécoise d'ethnologie (SQE) en 2017, ethnologiequebec.org/2017/09/les-retrouvailles-des-heritiers-de-medard-bourgault-unimmense-succes, et les animaliers Noël et Mario Guay ont reçu les hommages de la SQE lors de la Biennale de sculpture de Saint-Jean-Port-Joli en 2018, ethnologiequebec.org/2018/08/sculpteursanimaliers/, consultés le 23 juin 2020.

160. Mмv, Entrevue avec Pierre Bourgault par Jean-François Blanchette, 5 juin 2017.

161. La Presse, 18 mai 1932, p. 6, cité par Angéline Saint-Pierre, André Bourgault, op. cit., p. 36.

162. Derek Gill, «The modern artisans of St. Jean Port Joli », Los Angeles, California, Pace, juillet 1967, p. 3 et 58-61. 
sculpteur, 1897-1967 163 », qui fait un survol éloquent de l'œuvre des trois maîtres sculpteurs.

\section{Témoignage}

Terminons avec le témoignage d'un sculpteur qui raconte son apprentissage de la vie et de la sculpture avec Médard et Jean-Julien. Gilles-Jacques Cloutier (1948-2014) va à l'école de sculpture de Jean-Julien Bourgault de 1963 à 1965. Il raconte son parcours et ce que Jean-Julien et Médard Bourgault furent pour lui :

Moi, je n'avais pas le droit d'aller à l'école de sculpture parce que j'étais trop jeune. Ça prenait 16 ans, j'avais 15 ans. J'aimais pas ça chez nous parce que mon père me battait. J'ai trafiqué mon baptistère et avec l'aide de ma mère, je suis rentré à l'école de sculpture. Jean-Julien m'a pris sous son aile et il m'a montré à sculpter.

Dans le temps, dans les années 1960, c'était l'époque des hippies. D'ailleurs, c'est nous autres qui avons parti les hippies à Saint-Jean-Port-Joli. C'est nous qui avons commencé à avoir les cheveux longs, jusque-là, puis habillés avec des peaux de mouton. C'était la belle époque. Les petites filles couraient toutes après nous autres et nous, on courait après les petites filles...

En attendant que l'école ouvre à huit heures, j'étais à l'atelier de Médard tous les matins, pendant deux ans. Médard arrivait à 6 h 30. Il ouvrait sa porte et moi j'entrais. Médard s'assoyait, puis il roulait sa cigarette, puis il se berçait et fumait sa cigarette. Il me faisait juste un coup de tête en voulant dire "Bonjour ». Après ça, il se levait puis il sculptait ses belles femmes nues. Il les flattait parce qu'il était presque aveugle, et il fallait qu'il touche. Il touchait pour voir si le coup de ciseau qu'il avait donné était correct. Je trouvais ça merveilleux! Médard m'a aidé beaucoup, malgré qu'il ne parlait pas beaucoup.

Je n'avais pas d'argent quand j'ai commencé. J'étais marié, j'avais une épouse. Je n'avais pas les moyens de m'acheter du bois. Je prenais des bois d'épave que je trouvais sur la grève, comme Médard qui m'inspirait. Médard avait des beaux bois d'épave qu'il travaillait près de ses petits chalets. Je trouvais ça tellement beau et simple. Je me suis mis à travailler ça, à faire des visages que je voyais dans ça...

Mais là, il arrivait des gens chez moi. J'avais un petit atelier dans un coin de ma petite maison et le monde rentrait. Il est arrivé des gens d'Europe. C'est pas pour dire qu'ils sont snobs ou quoi que ce soit, mais ils ont plus d'éducation du côté mythologie. Ils s'exclamaient : "C'est Zeus, c'est Neptune, ça, c'est Éole! » - "C'est qui ça, Éole? » Je n'avais pas lu, moi là... Je ne suis pas allé à l'école longtemps, j'ai juste une septième année. Je ne connaissais pas la mythologie. J'ai commencé à acheter des livres et j'ai lu la mythologie grecque et romaine et, de là, j'ai découvert mon style. J'ai découvert ces dieux-là dans la

163. Yves Dubé, Forces, nº 4, hiver 1968, p. 30-41, 20 ill. 
sculpture. Tous des dieux de la mythologie, Éole, Morphée, Thanatos, Hypnos, tout ce qui se rapporte à la mort, tout ce qui se rapporte à la vie, comme le dieu des vents, Éole, le dieu de la mer, Neptune, les déesses comme Diane, la déesse de la chasse. J'ai découvert une passion. Je me levais le matin et j'avais le goût de vivre, j'avais le goût d'écrire.

Je faisais des dieux, là, puis les personnes rentraient, ils regardaient le visage et je te jure sur dix personnes, il y en avait une qui sortait avec une sculpture. C'était une belle récompense de voir les yeux des gens quand ils regardaient la sculpture. Les gens "shakaient". Ils frémissaient. C'était un peu comme si tu entends une chanson et que le poil te redresse sur les bras. Ils rentraient chez moi et, quand ils regardaient la sculpture, ils frémissaient. Je disais : "J'ai réussi ma sculpture. "Parce que j'exprimais des sentiments dans les yeux, dans les visages : c'était soit de la douleur, de la frayeur ou de la douceur. Je faisais des beaux visages de femmes, ou bien un diable, la gueule ouverte, qui veut manger le monde. Tout dépendant de la forme de ma bûche et de mon intérieur, ce que je ressentais cette journée-là. Mais j'avais la possibilité de réaliser, d'exprimer ce que beaucoup de gens n'ont pas : la sincérité. C'est plus l'émotion qu'autre chose! Le coup de ciseau, tout le monde l'a. Tu as beau savoir couper du bois, aller chercher un coup de ciseau. Mais si tu n'es pas capable de donner tes émotions, si tu n'es pas capable de faire ce que tu veux faire, tu ne seras jamais capable de le faire. Pour faire un visage, un sourire, les lèvres, les yeux d'une femme, il n'y a rien de plus difficile que de faire une belle femme. À Saint-Jean-Port-Joli, il n'y en a pas beaucoup qui sont capables. Faire des petites vieilles, des petits vieux, y sont capables en masse! Mais une belle jeune femme de 30 ans, 35-40 ans, cherchez-moi un sculpteur qui est capable d'en faire.

Avec ce que j'ai vécu dans mon enfance, c'est une forme de libération de toutes les souffrances. Parce que dans mes sculptures, là, tu voyais ma tristesse. Tu voyais mes joies. Tu voyais mes peines. Tu voyais la souffrance qu'on a vécue chez nous. Mais je n'ai pas de mauvais souvenirs. Moi, j'ai aimé mon métier. Je recommencerais ma vie demain matin. J'ai tellement aimé ma vie ! Le bord du fleuve, c'est de toute beauté! De toute beauté! Ah non, je n'ai pas de mauvais souvenirs! Les plus beaux souvenirs que j'ai, c'est Médard. Et des mauvais souvenirs, je n'en ai pas. Je n'ai pas de mauvais souvenirs!

Par contre, j'ai tellement de beaux souvenirs! J'ai tellement aimé ma vie. C'est tout beau et je recommencerais ma vie. Je dirais que mon plus beau souvenir, c'est Médard Bourgault. Premièrement, cet homme-là m'a tellement donné. Ce n'est pas possible! Le matin, j'avais froid. Il me donnait de la chaleur! Il me donnait son amour. Juste à me regarder et à se bercer dans sa chaise. Il fumait sa cigarette. Après ça, il commençait à travailler. Il ne travaillait pas vite, mais il travaillait longtemps! C'est mon plus beau souvenir : Médard Bourgault ${ }^{164}$.

164. Mmv 2007-0047, Entrevue de Gilles-Jacques Cloutier (1948-2014) par Martine Bélanger, 13 décembre 2007. 